\title{
Semirelativistic description of quasielastic neutrino reactions and superscaling in a continuum shell model
}

\author{
J. E. Amaro, ${ }^{1}$ M. B. Barbaro, ${ }^{2}$ J. A. Caballero, ${ }^{3}$ T. W. Donnelly, ${ }^{4}$ and C. Maieron ${ }^{5}$ \\ ${ }^{1}$ Departamento de Física Moderna, Universidad de Granada, Granada E-18071, Spain \\ ${ }^{2}$ Dipartimento di Fisica Teorica, Università di Torino and INFN, Sezione di Torino Via P. Giuria 1, I-10125 Torino, Italy \\ ${ }^{3}$ Departamento de Física Atómica, Molecular y Nuclear Universidad de Sevilla, Apdo. 1065, E-41080 Sevilla, Spain \\ ${ }^{4}$ Center for Theoretical Physics, Laboratory for Nuclear Science and Department of Physics, Massachusetts Institute of Technology, \\ Cambridge, Massachusetts 02139, USA \\ ${ }^{5}$ INFN, Sezione di Catania, Via Santa Sofia 64, I-95123 Catania, Italy
}

(Received 23 March 2005; published 9 June 2005)

\begin{abstract}
The so-called semirelativistic expansion of the weakly charged current in powers of the initial nucleon momentum is performed to describe charge-changing, quasielastic neutrino reactions $\left(v_{\mu}, \mu^{-}\right)$at intermediate energies. The quality of the expansion is tested by comparing it with the relativistic Fermi gas model using several choices of kinematics of interest for ongoing neutrino oscillation experiments. The new current is then implemented in a continuum shell model together with relativistic kinematics to investigate the scaling properties of $\left(e, e^{\prime}\right)$ and $\left(v_{\mu}, \mu^{-}\right)$cross sections.
\end{abstract}

DOI: 10.1103/PhysRevC.71.065501

PACS number(s): 25.30.Pt, 25.30.Fj, 24.10.Jv, 21.60.Cs

\section{INTRODUCTION}

The importance of neutrino-induced reactions in nuclei has been stressed in connection with the neutrino oscillation experiments performed by the KARMEN and LSND Collaborations [1-8]. In experiments of this type, relatively low $v_{e}$ or $v_{\mu}$ energies are involved (at most a few hundreds of $\mathrm{MeV}$ ), and so the nuclear excitations involved can be described by standard nonrelativistic models of the reaction including the relevant machinery random-phase-approximation (RPA) correlations, large-basis shell models, $\Delta$-hole excitations, final-state interactions, etc. for this energy regime, where in particular giant resonances may play an important role [9-16].

However, when passing to the ongoing and next generation of neutrino experiment, MiniBooNE, K2K/T2K, MINOS, $\mathrm{NO} v \mathrm{~A}$, and MINER $v \mathrm{~A}$ [17-22], the neutrino beam energies increase to the $\mathrm{GeV}$ level, and typically large energies and momenta are transferred to the nucleus. For these kinematics, relativity is important and nonrelativistic models of the reaction such as those listed above are bound to fail unless the relevant relativistic ingredients are included.

First of all, the use of relativistic kinematics is required and must be implemented in the model. From quasielastic (QE) electron scattering studies we know that a good approximation to the correct kinematics consists in the substitution

$$
\lambda \longrightarrow \lambda(1+\lambda),
$$

with $\lambda=\omega / 2 m_{N}$, where $\omega$ is the energy transfer and $m_{N}$ the nucleon mass. This substitution can easily be performed in all places in the calculation - except in the nucleon form factors, where the correct value of momentum transfer $Q^{\mu}=(\omega, \mathbf{q})$ must be used. Second, a good approximation to the current matrix elements is required. The current is traditionally obtained from the fully relativistic one by some expansion procedure, usually through the Foldy-Wouthuysen (FW) transformation, which is valid for small momenta compared to the nucleon mass. Within this procedure, relativistic effects were studied for neutrino energies up to $300 \mathrm{MeV}$ in [23], showing that a good agreement is obtained between fully relativistic and non relativistic Fermi gas calculations, when terms up to order $\left(q / m_{N}\right)^{3}$ are included in the latter. Similar results were obtained at larger energies, for the scattering of atmospheric neutrinos from oxygen [24], where, however, it must be noted that because of the steep decrease of the atmospheric neutrino spectrum at large energies (see, for example, [25]), the most important contributions to the process correspond to relatively small energy transfers. Obviously, the Foldy-Wouthuysen expansion is not applicable to the high values of the momentum transfer $q$ which are of interest to the new experiments, i.e., for values around $1 \mathrm{GeV} / c$. In this case, a different expansion procedure must be performed in which $q$ and $\omega$ can be arbitrarily large.

Thus, the first goal of this paper is to develop an approximation to the nuclear charged current (CC) that accounts for specific relativistic effects relevant to intermediate-energy quasielastic neutrino reactions. The $\mathrm{CC}$ is obtained here through an expansion that only requires the momentum of the initial nucleon to be small, while it treats exactly the dependence on $(\omega, \mathbf{q})$. Moreover, the resulting semirelativistic (SR) current is simple enough to be easily implemented in already existing nonrelativistic models of $\left(v_{l}, l^{-}\right)$and $\left(\bar{v}_{l}, l^{+}\right)$ reactions, where $l=e$ or $\mu$. It is presented here as an extension of the electromagnetic and weak-neutral current expansion originally derived in [26] and that in recent years has been widely tested and applied in several Collaborations to describe a wide variety of inclusive and exclusive electron scattering observables for intermediate energies and excitations in the vicinity of the QE peak [27-37]. Extensions of the SR expansion to meson-exchange currents have also been developed [38,39], and a detailed description of their application to two-body currents can be found in a recent review article [40]. 
In this paper, we apply the SR model to neutrino-induced reactions, for the first time. Apart from the different isospin dependence, the SR expansion coincides essentially with that of the weak neutral current performed in [26]. The only difference is that in the present reaction, we have to include the time component of the axial-vector current. That component was not considered in [26] because it does not contribute significantly to parity-violating electron scattering.

We check the quality of the SR expansion in the context of the relativistic Fermi gas (RFG) model. This is a very convenient model for our purposes, since it is fully relativistic and simple enough to be solved exactly. Also this model is capable of getting the basic size and shape of the QE $\left(e, e^{\prime}\right)$ cross section. Thus we will show that starting from the nonrelativistic Fermi gas, performing the replacement in Eq. (1) in the kinematics, and implementing the new SR charged current, we reproduce basically the RFG results for $\left(v_{\mu}, \mu^{-}\right)$reactions. This "relativizing" procedure can easily be extrapolated to more sophisticated finite nuclei models of the reaction. The result will at least reproduce appropriately the allowed kinematical region and the relevant relativistic content of the current operator. Of course, features related to the relativistic aspects of the dynamics cannot be accounted for by our procedure. In context we note that studies of relativistic nuclear dynamics in charged and neutral-current neutrino-nucleus QE scattering have already been presented in some previous work [41-45]. In these studies, a basic focus was to analyze the effects introduced by various descriptions of the final nucleon relativistic states upon the integrated cross sections.

We illustrate the relativizing procedure by applying it to the continuum shell model (CSM), i.e., nucleons in a mean field taken here to be a Woods-Saxon potential. We use the same (real) potential for initial and final states in order to maintain orthogonality between nuclear states. In this way, we extend the SR shell model of [26] to neutrino reactions at the QE peak. Since the use of relativistic kinematics is potentially equivalent to solving a Klein-Gordon equation, the present SR continuum shell model includes some aspects of a relativistic mean field.

With such a model, we are in a position to fulfill the second goal of this paper, which is to investigate superscaling properties of both $\left(e, e^{\prime}\right)$ and $\left(v_{\mu}, \mu^{-}\right)$inclusive cross sections at the $\mathrm{QE}$ peak for intermediate energies, namely, the degree to which one finds that the reduced cross sections are independent of the momentum transfer (scaling of the first kind) or the nuclear species (scaling of the second kind) or both (superscaling). Exhaustive analyses of the $\left(e, e^{\prime}\right)$ world data and explorations of various aspects of their scaling properties have been performed in [46-52]. In particular, in recent work [53], the approach has been extended to the $\Delta$ peak, allowing one to construct a semiempirical model based on scaling which is very successful in describing the experimental $\left(e, e^{\prime}\right)$ cross section up to the $\Delta$ peak for high energies. This model allowed us in [53] to generate predictions for the $\left(v_{\mu}, \mu^{-}\right)$cross section, under the reasonable hypothesis that it presents, for high energies, scaling properties similar to those of the $\left(e, e^{\prime}\right)$ cross section. This hypothesis is true by construction in the RFG and can be also demonstrated at least for the conventional plane-wave impulse approximation (PWIA) in the high $q$ limit. ${ }^{1}$ However, when distortion of the ejected nucleon is present, a general proof of scaling cannot be provided, while for lower energies, it is clear that the axial-vector and vector matrix elements are renormalized differently in the nuclear medium because of RPA correlations, and this can obviously modify the scaling properties of the neutrino cross section. For higher energies, one expects that nuclear effects such as from RPA correlations are less important than at low energies. In this work, we use the SR shell model to investigate the degree of violation of the scaling hypothesis for the QE peak region within the distorted-wave impulse approximation (DWIA), where the distortion of the ejected nucleon is described with a real potential. We perform this study in two steps. First we focus on the $\left(e, e^{\prime}\right)$ cross section and study the scaling properties of the separate response functions. Once the superscaling has been verified for electromagnetic processes, we are able to reconstruct the $\left(v_{\mu}, \mu^{-}\right)$cross section from the $\left(e, e^{\prime}\right)$ one using the scaling hypothesis and compare it with the one computed directly using the SR shell model. Thus, at least within the context of the SR shell model discussed in the present work, following this procedure we shall be able to check the consistency of our approach and quantify the degree to which scale breaking effects are expected to enter. As we shall see below, there appears to be very little impact from this source of scale breaking on the scaling approach used in [53] to predict neutrino-induced cross sections.

The paper is organized in the following way. In Sec. II we begin with a brief review of the general formalism for neutrino scattering and present the expansion of the CC. We particularize the formalism for the shell model and introduce the general multipole expansion of the responses, with some details on the derivation of the Coulomb multipoles of the axial-vector current placed in Appendix A. We also provide the expressions for the factorized PWIA in Appendix B. In Sec. III, we present results for the $\left(v_{\mu}, \mu^{-}\right)$reaction for several choices of kinematics and several nuclei of interest. We first perform an analysis of the quality of the relativizing procedure by comparison with the RFG for the relevant kinematics (the RFG model is further discussed in Appendix C.) The quality of the various components of the SR current can be checked separately by examining the individual response functions that contribute to the process. We then focus on the SR shell model and perform the scaling analysis of the longitudinal and transverse electromagnetic responses as functions of the momentum transfer for various nuclei. We apply the scaling hypothesis to reconstruct the $\left(v_{\mu}, \mu^{-}\right)$cross section starting from the electromagnetic scaling function and compare, our findings with the shell model results. Finally, in Sec. IV we present our conclusions.

\section{FORMALISM}

In this section, we briefly present the basic formalism for neutrino-induced reactions. Some of the previous approaches

\footnotetext{
${ }^{1}$ However, this is broken to some degree by the relativistic PWIA, where factorization no longer obtains.
} 
to the general formalism for these reactions can be found in [41,54-57].

\section{A. Charge-changing neutrino cross section}

Here we focus on the particular case of $\left(v_{\mu}, \mu^{-}\right)$, while the cases of antineutrinos or other lepton species can be easily obtained with obvious changes. The four momenta of the incident neutrino and detected muon are $k^{\mu}=(\epsilon, \mathbf{k})$ and $k^{\prime \mu}=\left(\epsilon^{\prime}, \mathbf{k}^{\prime}\right)$, respectively. The four-momentum transfer is $Q^{\mu}=k^{\mu}-k^{\prime \mu}=(\omega, \mathbf{q})$. We use a coordinate system with the $z$ axis pointing along $\mathbf{q}$ and the $x$ axis along the transverse component of the incident momentum, i.e., $\mathbf{k}^{\perp}=\mathbf{k}-\frac{1}{q^{2}}(\mathbf{k}$. q)q. We follow the formalism of [53], where the cross section is written as

$$
\frac{d \sigma}{d \Omega^{\prime} d \epsilon^{\prime}}=\sigma_{0} \mathcal{F}_{+}^{2}
$$

with

$$
\sigma_{0}=\frac{G^{2} \cos ^{2} \theta_{c}}{2 \pi^{2}} k^{\prime} \epsilon^{\prime} \cos ^{2} \frac{\tilde{\theta}}{2}
$$

Here $G=1.166 \times 10^{-11} \mathrm{MeV}^{-2}$ is the Fermi constant, $\theta_{c}$ is the Cabibbo angle, $\cos \theta_{c}=0.975$, and the angle $\tilde{\theta}$ is defined as

$$
\tan ^{2} \frac{\tilde{\theta}}{2}=\frac{\left|Q^{2}\right|}{\left(\epsilon+\epsilon^{\prime}\right)^{2}-q^{2}}
$$

with $Q^{2}=\omega^{2}-q^{2}<0$. The nuclear structure information is contained in $\mathcal{F}_{+}^{2}$, defined by

$$
\mathcal{F}_{+}^{2}=\widehat{V}_{\mathrm{CC}} R_{\mathrm{CC}}+2 \widehat{V}_{\mathrm{CL}} R_{\mathrm{CL}}+\widehat{V}_{\mathrm{LL}} R_{\mathrm{LL}}+\widehat{V}_{T} R_{T}+2 \widehat{V}_{T^{\prime}} R_{T^{\prime}},
$$

where the kinematical factors $\widehat{V}_{K}$ coming from the leptonic tensor are defined by

$$
\begin{aligned}
& \widehat{V}_{\mathrm{CC}}=1-\delta^{2} \tan ^{2} \frac{\tilde{\theta}}{2}, \\
& \widehat{V}_{\mathrm{CL}}=\frac{\omega}{q}+\frac{\delta^{2}}{\rho^{\prime}} \tan ^{2} \frac{\tilde{\theta}}{2}, \\
& \widehat{V}_{\mathrm{LL}}=\frac{\omega^{2}}{q^{2}}+\left(1+\frac{2 \omega}{q \rho^{\prime}}+\rho \delta^{2}\right) \delta^{2} \tan ^{2} \frac{\tilde{\theta}}{2}, \\
& \widehat{V}_{T}=\tan ^{2} \frac{\tilde{\theta}}{2}+\frac{\rho}{2}-\frac{\delta^{2}}{\rho^{\prime}}\left(\frac{\omega}{q}+\frac{1}{2} \rho \rho^{\prime} \delta^{2}\right) \tan ^{2} \frac{\tilde{\theta}}{2}, \\
& \widehat{V}_{T^{\prime}}=\frac{1}{\rho^{\prime}}\left(1-\frac{\omega \rho^{\prime}}{q} \delta^{2}\right) \tan ^{2} \frac{\tilde{\theta}}{2} .
\end{aligned}
$$

In Eqs. (6)-(10), following [53], we have defined

$$
\begin{aligned}
\delta & =\frac{m^{\prime}}{\sqrt{\left|Q^{2}\right|}}, \\
\rho & =\frac{\left|Q^{2}\right|}{q^{2}}, \\
\rho^{\prime} & =\frac{q}{\epsilon+\epsilon^{\prime}} .
\end{aligned}
$$

Note that the only dependence on the muon mass $m^{\prime}$ is contained in the $\delta$ coefficient.

Finally, the weak response functions are given by

$$
\begin{aligned}
R_{\mathrm{CC}} & =W^{00}, \\
R_{\mathrm{CL}} & =-\frac{1}{2}\left(W^{03}+W^{30}\right), \\
R_{\mathrm{LL}} & =W^{33}, \\
R_{T} & =W^{11}+W^{22}, \\
R_{T^{\prime}} & =-\frac{i}{2}\left(W^{12}-W^{21}\right),
\end{aligned}
$$

in terms of the inclusive hadronic tensor [54]

$$
W^{\mu \nu}(q, \omega)=\overline{\sum_{f i}} \delta\left(E_{f}-E_{i}-\omega\right)\left\langle f\left|J^{\mu}(Q)\right| i\right\rangle^{*}\left\langle f\left|J^{\nu}(Q)\right| i\right\rangle .
$$

In Eq. (19), $J^{\mu}(Q)$ is the hadronic CC current operator, to be specified below, and a sum over final states and an average over initial spin is assumed.

\section{B. Semirelativistic charge-changing current}

We begin with the basic relativistic charged weak current of the nucleon, $j^{\mu}=j_{V}^{\mu}-j_{A}^{\mu}$. In this work, we employ only the standard model of electroweak interactions at tree level and thus, for example, do not include radiative corrections or contributions from second-class currents (see [54]). We use the conventions of [58]. The vector and axial-vector currents are given by

$$
\begin{gathered}
j_{V}^{\mu}\left(\mathbf{p}^{\prime}, \mathbf{p}\right)=\bar{u}\left(\mathbf{p}^{\prime}\right)\left[2 F_{1}^{V} \gamma^{\mu}+i \frac{F_{2}^{V}}{m_{N}} \sigma^{\mu \nu} Q_{v}\right] u(\mathbf{p}), \\
j_{A}^{\mu}\left(\mathbf{p}^{\prime}, \mathbf{p}\right)=\bar{u}\left(\mathbf{p}^{\prime}\right)\left[G_{A} \gamma^{\mu}+G_{P} \frac{Q^{\mu}}{2 m_{N}}\right] \gamma^{5} u(\mathbf{p}),
\end{gathered}
$$

where for the isovector nucleon form factors $F_{1,2}^{V}=\left(F_{1,2}^{p}-\right.$ $\left.F_{1,2}^{n}\right) / 2$ we use the Galster parametrization [59], and $u(\mathbf{p})$ is the free Dirac spinor of the nucleon. The axial-vector and pseudoscalar form factors are parametrized as

$$
\begin{aligned}
G_{A} & =\frac{g_{A}}{1-Q^{2} / M_{A}^{2}}, \\
G_{P} & =\frac{4 m_{N}^{2}}{m_{\pi}^{2}-Q^{2}} G_{A},
\end{aligned}
$$

with $g_{A}=1.26, M_{A}=1032 \mathrm{MeV}$.

The SR approximation to this current is then obtained by inserting the appropriate free spinors $u(\mathbf{p}), u\left(\mathbf{p}^{\prime}\right)$, and $\gamma$ matrices, and performing an expansion in powers of $\boldsymbol{\eta} \equiv \mathbf{p} / m_{N}$ to first order. The procedure was developed in $[26,38,40]$ for the electromagnetic and transverse neutral, vector, and axial-vector currents and is based on the fact that inside the nucleus $|\boldsymbol{\eta}|$ is a good expansion parameter, the characteristic dimensionless nuclear scale being $\eta_{F}=k_{F} / m_{N} \simeq 1 / 4$. We exploit QE kinematics, while further reasonable simplifications are eventually needed in order to 
arrive at simple expressions that are easily implementable in traditional nonrelativistic calculations. For the vector current, we use the following SR approximation:

$$
\begin{aligned}
& J_{V}^{0}=\xi_{0}+i \xi_{0}^{\prime}(\boldsymbol{\kappa} \times \boldsymbol{\eta}) \cdot \boldsymbol{\sigma} \\
& \mathbf{J}_{V}^{\perp}=\xi_{1} \boldsymbol{\eta}^{\perp}+i \xi_{1}^{\prime} \boldsymbol{\sigma} \times \boldsymbol{\kappa},
\end{aligned}
$$

where

$$
\begin{array}{ll}
\xi_{0}=\frac{\kappa}{\sqrt{\tau}} 2 G_{E}^{V}, \quad \xi_{0}^{\prime}=\frac{2 G_{M}^{V}-G_{E}^{V}}{\sqrt{1+\tau}}, \\
\xi_{1}^{\prime}=2 G_{M}^{V} \frac{\sqrt{\tau}}{\kappa}, \quad \xi_{1}=2 G_{E}^{V} \frac{\sqrt{\tau}}{\kappa},
\end{array}
$$

and use has been made of the dimensionless variables $\kappa=$ $\mathbf{q} / 2 m_{N}$ and $\tau=\kappa^{2}-\lambda^{2}$.

From vector current conservation, the longitudinal component is given by $J_{V}^{3}=\frac{\lambda}{\kappa} J_{V}^{0}$. Note that in Eqs. (24) and (25), the various terms making up the current are similar to the ones that can be found in traditional nonrelativistic expansions commonly used for the charged current (see, for instance, [16]), except for the $\kappa$ - and $\tau$-dependent factors, $\xi_{i}, \xi_{i}^{\prime}$, that provide the required relativistic behavior. In $J_{V}^{0}$ we include the first-order $O(\eta)$ contribution. This spin-orbit term is proportional to the operator $(\kappa \times \boldsymbol{\eta}) \cdot \boldsymbol{\sigma}$ and is of some importance for high $q$ values. On the other hand, the transverse component $\mathbf{J}_{V}^{\perp}$ is the sum of the usual magnetization $(\sigma \times \kappa)$ piece plus a first-order term, the convection term which is proportional to $\eta^{\perp}$, that gives in general a very small ${ }^{2}$ contribution to the cross section for high $q[26,28]$.

In the case of the axial-vector sector, only the transverse component of the weak neutral current was expanded in [26]. We use the following version of the corresponding SR current

$$
\mathbf{J}_{A}^{\perp}=\zeta_{1}^{\prime} \boldsymbol{\sigma}^{\perp}, \quad \zeta_{1}^{\prime}=\sqrt{1+\tau} G_{A} .
$$

Note that we have neglected the terms of order $\eta$ since, as we shall show in the next section (and also demonstrated in [26] in the context of parity-violating electron scattering), they are small and add unnecessary complications to the shell model calculation. They can be safely neglected for our purposes. Remarkably, the factor $\sqrt{1+\tau}$ in Eq. (28) already accounts for relativistic effects in this current (see [26] for the full expansion of this current to first order in $\eta$ ).

We are left with the 0 and $z$ components of the axial-vector current. Their SR expressions are presented here for the first time. Using a notation reminiscent of that used in Appendix A of [26], we write them to first order in $\eta$ as

$$
\begin{aligned}
& J_{A}^{0}=\zeta_{0}^{\prime} \boldsymbol{\kappa} \cdot \boldsymbol{\sigma}+\zeta_{0}^{\prime \prime} \eta^{\perp} \cdot \boldsymbol{\sigma}, \\
& J_{A}^{z}=\zeta_{3}^{\prime} \boldsymbol{\kappa} \cdot \boldsymbol{\sigma}+\zeta_{3}^{\prime \prime} \eta^{\perp} \cdot \sigma,
\end{aligned}
$$

\footnotetext{
${ }^{2}$ Note that $\boldsymbol{\eta}=\mathbf{p} / m_{N}$ is essentially the velocity $\mathbf{v}$ of the initial struck nucleon in units of $c$.
}

where

$$
\begin{aligned}
& \zeta_{0}^{\prime}=\frac{1}{\sqrt{\tau}} \frac{\lambda}{\kappa} G_{A}^{\prime}, \quad \zeta_{0}^{\prime \prime}=\frac{\kappa}{\sqrt{\tau}}\left[G_{A}-\frac{\lambda^{2}}{\kappa^{2}+\kappa \sqrt{\tau(\tau+1)}} G_{A}^{\prime}\right], \\
& \zeta_{3}^{\prime}=\frac{1}{\sqrt{\tau}} G_{A}^{\prime}, \quad \zeta_{3}^{\prime \prime}=\frac{\lambda}{\sqrt{\tau}}\left[G_{A}-\frac{\kappa}{\kappa+\sqrt{\tau(\tau+1)}} G_{A}^{\prime}\right],
\end{aligned}
$$

and we have introduced the following combination of axialvector and pseudoscalar form factors

$$
G_{A}^{\prime}=G_{A}-\tau G_{P} .
$$

For these components of the axial-vector current, Eqs. (29) and (30), we have performed the expansion to first order in $\eta$. The first-order axial-convective term is proportional to $\sigma \cdot \eta^{\perp}$, and, as for the spin-orbit and convection terms, only the perpendicular velocity $\eta^{\perp}$ appears. As we shall show below, for the kinematics of interest in this work, the $G_{A}^{\prime}$ form factor which drives the zeroth-order terms turns out to be small because of cancellations in Eq. (33) between $G_{A}$ and $\tau G_{P}$. In such cases, the $O(\eta)$ term is dominant in these current components.

\section{The continuum shell model}

In this work, we restrict our attention to the closed-shell ${ }^{3}$ nuclei ${ }^{12} \mathrm{C},{ }^{16} \mathrm{O}$, and ${ }^{40} \mathrm{Ca}$, which we describe in a continuum shell model (CSM). Thus the present model does not include nuclear correlations. Such effects are important for low energy, in particular in the axial responses, and have been estimated, for instance, in the RPA approach of Ref. [15], but are expected to be smaller at the $\mathrm{GeV}$ energies considered in this paper. The initial state $|i\rangle$ appearing in the hadronic tensor, Eq. (19), is described as a Slater determinant representing the uncorrelated nuclear core with all shells occupied. Since we are working in the impulse approximation, the final states are particle-hole excitations coupled to total angular momentum $J$, namely, $|f\rangle=\left|\left(p h^{-1}\right) J\right\rangle$. The single hole $|h\rangle=\left|\epsilon_{h} l_{h} j_{h}\right\rangle$ and particle $|p\rangle=\left|\epsilon_{p} l_{p} j_{p}\right\rangle$ wave functions are obtained by solving the Schrödinger equation with a Woods-Saxon potential

$$
V(r)=-V_{0} f\left(r, R_{0}, a_{0}\right)+\frac{V_{l s}}{m_{\pi}^{2} r} \frac{d f\left(r, R_{0}, a_{0}\right)}{d r} \mathbf{l} \cdot \boldsymbol{\sigma}+V_{C}(r),
$$

where

$$
f(r, R, a)=\frac{1}{1+e^{(r-R) / a}},
$$

and $V_{C}(r)$ is the Coulomb potential of a charged sphere of charge $Z-1$ and radius $R_{0}$ (it is equal to zero for neutrons). The parameters of the potential are fitted to the experimental energies of the valence shells and are given in Table I.

\footnotetext{
${ }^{3}$ By shell we mean a subshell with quantum numbers $(n l j)$.
} 
TABLE I. Woods-Saxon potential parameters for protons $p$ and neutrons $n$. Units are $\mathrm{MeV}$ for $V_{i}$, and fm for $a_{0}$ and $r_{0}$. The reduced radius parameter $r_{0}$ is defined by $R_{0}=r_{0} A^{1 / 3}$.

\begin{tabular}{lcccccc}
\hline \hline & $V_{0}^{p}$ & $V_{L S}^{p}$ & $V_{0}^{n}$ & $V_{L S}^{n}$ & $r_{0}$ & $a_{0}$ \\
\hline${ }^{12} \mathrm{C}$ & 62.0 & 3.20 & 60.00 & 3.15 & 1.25 & 0.57 \\
${ }^{16} \mathrm{O}$ & 52.5 & 7.00 & 52.50 & 6.54 & 1.27 & 0.53 \\
${ }^{40} \mathrm{Ca}$ & 57.5 & 11.11 & 55.00 & 8.50 & 1.20 & 0.53 \\
\hline \hline
\end{tabular}

In the shell model, the energy transfer is computed as the difference between the (nonrelativistic) single-particle energies of particle and hole $\omega=\epsilon_{p}-\epsilon_{h}$. The relativistic kinematics are taken into account by the substitution

$$
\epsilon_{p} \rightarrow \epsilon_{p}\left(1+\epsilon_{p} / 2 m_{N}\right)
$$

as the eigenvalue of the Schrödinger equation for the particle; cf., Eq. (1).

Since the nuclear states have good angular momentum, it is convenient for the shell model calculation to perform a multipole expansion of the components of the current operator in terms of the usual Coulomb $(C)$, longitudinal $(L)$, transverse electric $(E)$, and transverse magnetic $(M)$ operators [26,54,60], defined by

$$
\begin{aligned}
& \hat{C}_{J 0}(q)=\int d^{3} r j_{J}(q r) Y_{J 0}(\hat{\mathbf{r}}) J_{0}(\mathbf{r}), \\
& \hat{L}_{J 0}(q)=\frac{i}{q} \int d^{3} r \nabla\left[j_{J}(q r) Y_{J 0}(\hat{\mathbf{r}})\right] \cdot \mathbf{J}(\mathbf{r}), \\
& \hat{E}_{J m}(q)=\frac{1}{q} \int d^{3} r \nabla \times\left[j_{J}(q r) \mathbf{Y}_{J J m}(\hat{\mathbf{r}})\right] \cdot \mathbf{J}(\mathbf{r}), \\
& \hat{M}_{J m}(q)=\int d^{3} r j_{J}(q r) \mathbf{Y}_{J J m}(\hat{\mathbf{r}}) \cdot \mathbf{J}(\mathbf{r}),
\end{aligned}
$$

where $j_{J}(q r)$ is a spherical Bessel function and $\mathbf{Y}_{J J m}(\hat{\mathbf{r}})$ is a vector spherical harmonic.

The nuclear response functions are then written as

$$
\begin{aligned}
& R_{\mathrm{CC}}=4 \pi \sum_{\alpha}\left(\left|C_{\alpha}^{V}\right|^{2}+\left|C_{\alpha}^{A}\right|^{2}\right) \\
& R_{\mathrm{CL}}=2 \pi \sum_{\alpha}\left(C_{\alpha}^{V *} L_{\alpha}^{V}+C_{\alpha}^{V} L_{\alpha}^{V *}+C_{\alpha}^{A *} L_{\alpha}^{A}+C_{\alpha}^{A} L_{\alpha}^{A *}\right) \\
& R_{\mathrm{LL}}=4 \pi \sum_{\alpha}\left(\left|L_{\alpha}^{V}\right|^{2}+\left|L_{\alpha}^{A}\right|^{2}\right) \\
& R_{T}=4 \pi \sum_{\alpha}\left(\left|E_{\alpha}^{V}\right|^{2}+\left|M_{\alpha}^{V}\right|^{2}+\left|E_{\alpha}^{A}\right|^{2}+\left|M_{\alpha}^{A}\right|^{2}\right) \\
& R_{T^{\prime}}=2 \pi \sum_{\alpha}\left(E_{\alpha}^{V *} M_{\alpha}^{A}+E_{\alpha}^{V} M_{\alpha}^{A *}+E_{\alpha}^{A *} M_{\alpha}^{V}+E_{\alpha}^{A} M_{\alpha}^{V *}\right),
\end{aligned}
$$

where in the above sums over $\alpha$, the particle energy $\epsilon_{p}=$ $\epsilon_{h}+\omega$ is modified according to Eq. (36), and we use the index $\alpha$ to label the quantum numbers $\alpha=\left(h, l_{p}, j_{p}, J\right)$. Moreover, the $C, L, E$, and $M$ multipoles of the vector and axial-vector currents are defined by the reduced matrix elements of the corresponding operators

$$
\begin{aligned}
C_{\alpha}^{V}+i C_{\alpha}^{A} & =\left\langle f\left\|\hat{C}_{J}(q)\right\| i\right\rangle, \\
L_{\alpha}^{V}+i L_{\alpha}^{A} & =\left\langle f\left\|\hat{L}_{J}(q)\right\| i\right\rangle, \\
E_{\alpha}^{V}+i E_{\alpha}^{A} & =\left\langle f\left\|\hat{E}_{J}(q)\right\| i\right\rangle, \\
-i M_{\alpha}^{V}-M_{\alpha}^{A} & =\left\langle f\left\|\hat{M}_{J}(q)\right\| i\right\rangle .
\end{aligned}
$$

These reduced matrix elements are given in [26] for the vector and axial-vector transverse operators and in [60] for the leading-order longitudinal axial-vector component. In the present paper, we add the first-order convective term of the axial-vector current $\eta^{\perp} \cdot \sigma$ appearing in Eqs. (29) and (30). The $C_{\alpha}^{A}$ and $L_{\alpha}^{A}$ multipoles of this new term are presented in Appendix A.

The sums over $\alpha$ in Eqs. (41)-(45) are infinite and have to be truncated in the calculation once convergence is reached. In our approach, the number of multipoles is determined by the maximum, of the total angular momentum $J_{\max }$. We fix this quantity by computing the response functions setting the potential in the final state to zero and comparing with the factorized PWIA (see Appendix B for details). The number $J_{\max }$ increases with $q$ and with the number of nucleons $A$. In the next section up to 41 multipoles have to be summed for the case where $q=1.5 \mathrm{GeV} / c$.

\section{RESULTS}

\section{A. Test of the SR approach}

The quality of the SR expansion of the charged current is illustrated in Figs. 1-3. There we show the separate vector, axial-vector, and total response functions of ${ }^{12} \mathrm{C}$ for three values of the momentum transfer, $q=0.5,1$, and $1.5 \mathrm{GeV} / c$. The Fermi momentum is chosen to be $k_{F}=$ $220 \mathrm{MeV} / c$. We show the separate response functions instead of cross sections because in these functions we can appreciate better the contribution of the various current components and the behavior of the SR approximation as a function of the momentum and energy transfers. This can be seen in the figures by comparing the solid lines, representing the RFG result (exact relativistic answer) with the dashed lines, corresponding to the semirelativistic Fermi gas (SRFG). This last model is obtained by implementing the SR expansion of the current in a nonrelativistic Fermi gas, although with relativistic kinematics. The accord of the two models is almost perfect for the three values of $q$ considered. In Fig. 1, we only show $R_{\mathrm{CC}}^{V}$ and $R_{T}^{V}$, since $R_{\mathrm{CL}}^{V}$ and $R_{\mathrm{LL}}^{V}$ are related to $R_{\text {CC }}^{V}$ by current conservation. In Fig. 2, we give instead the three response functions, since the axial-vector current is not conserved. Finally, in Fig. 3, we display the sums of Figs. 1 and 2 and also the interference response $R_{T^{\prime}}$.

In Figs. 1-3 we also plot (as dotted lines) the CSM responses, computed using the relativizing procedure. For comparison, we show the PWIA results obtained by setting the potential in the final state to zero (or, equivalently, by integration of the factorized exclusive responses, as shown in 

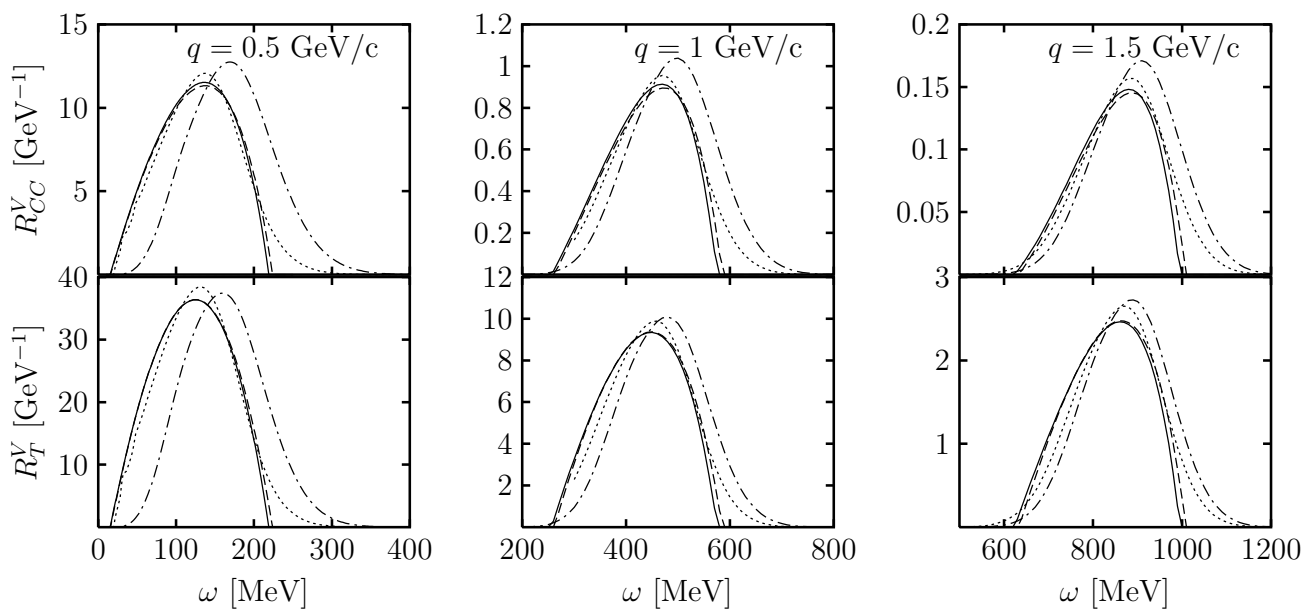

FIG. 1. Vector response functions of ${ }^{12} \mathrm{C}$ for three values of the momentum transfer. Solid lines: RFG with $k_{F}=220 \mathrm{MeV} / c$. Dashed lines: SRFG. Dotted: CSM. Dot-dashed: PWIA.

Appendix B). The CSM response functions are quantitatively similar in magnitude to the RFG and are centered approximately around the same value of $\omega$. The major discrepancy between the two models is found for the axial-vector $R_{\mathrm{CC}}^{A}, R_{\mathrm{CL}}^{A}$, and $R_{\mathrm{LL}}^{A}$ response functions, where the CSM responses are slightly larger in magnitude than the RFG ones. These responses are in general small compared with the corresponding vector responses. This is a consequence of the suppression of the axial-vector current in the longitudinal channel becasue of the small value of the form factor $G_{A}^{\prime}$, defined in Eq. (33), for these kinematics. In fact, from the definition in Eq. (23) of the pseudoscalar form factor, one has

$$
G_{A}^{\prime}=\left(1-\frac{Q^{2}}{Q^{2}-m_{\pi}^{2}}\right) G_{A} .
$$

At the intermediate energies of interest, $\left|Q^{2}\right| \gg m_{\pi}^{2}$, and hence the factor inside the parenthesis in Eq. (50) is also small, of order $O\left(m_{\pi}^{2} / Q^{2}\right)$. In this situation the first-order, axial-vector convective term of the current, which is proportional to $\eta^{\perp} \cdot \sigma$
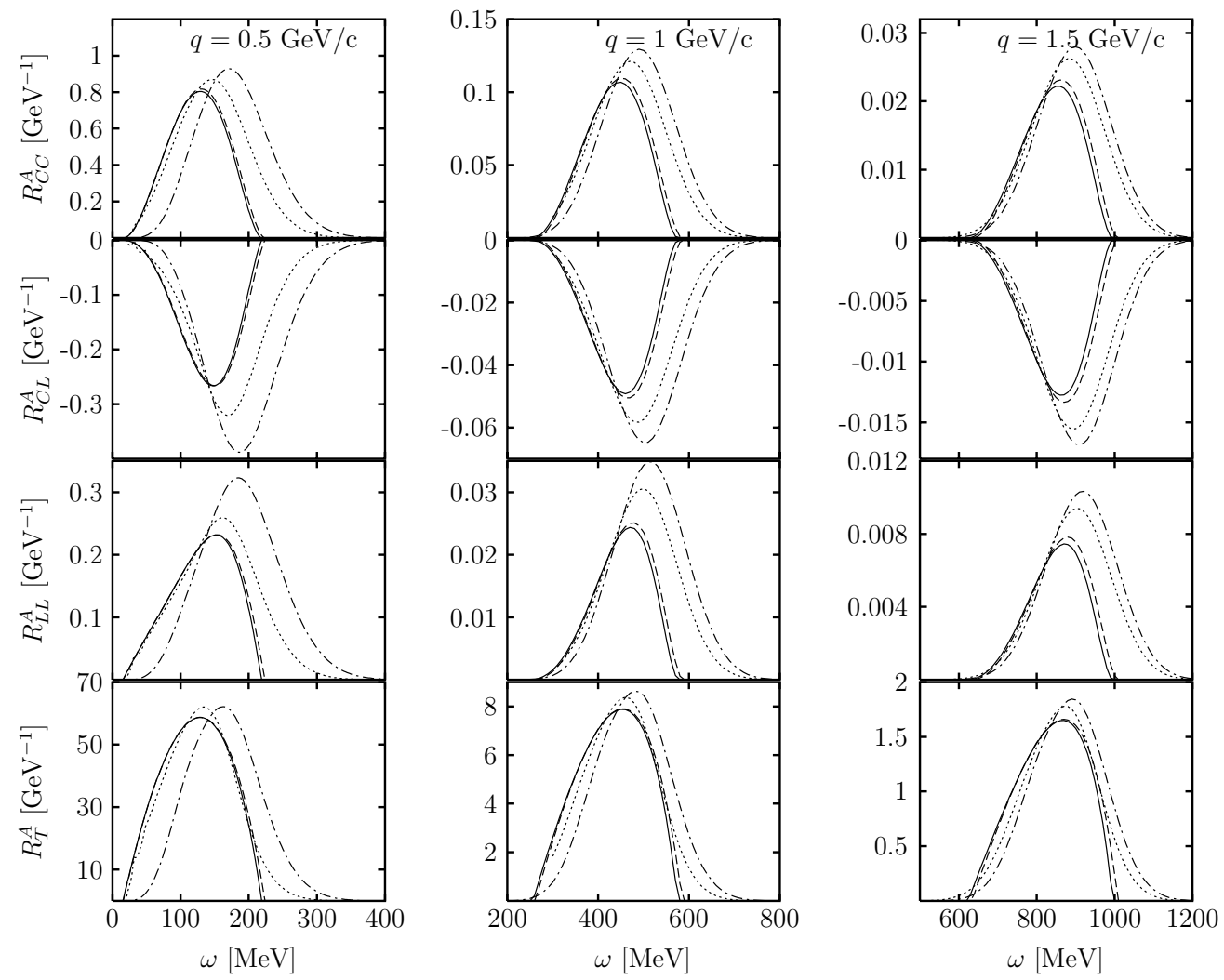

FIG. 2. Same as Fig. 1, but for the axial-vector responses. 

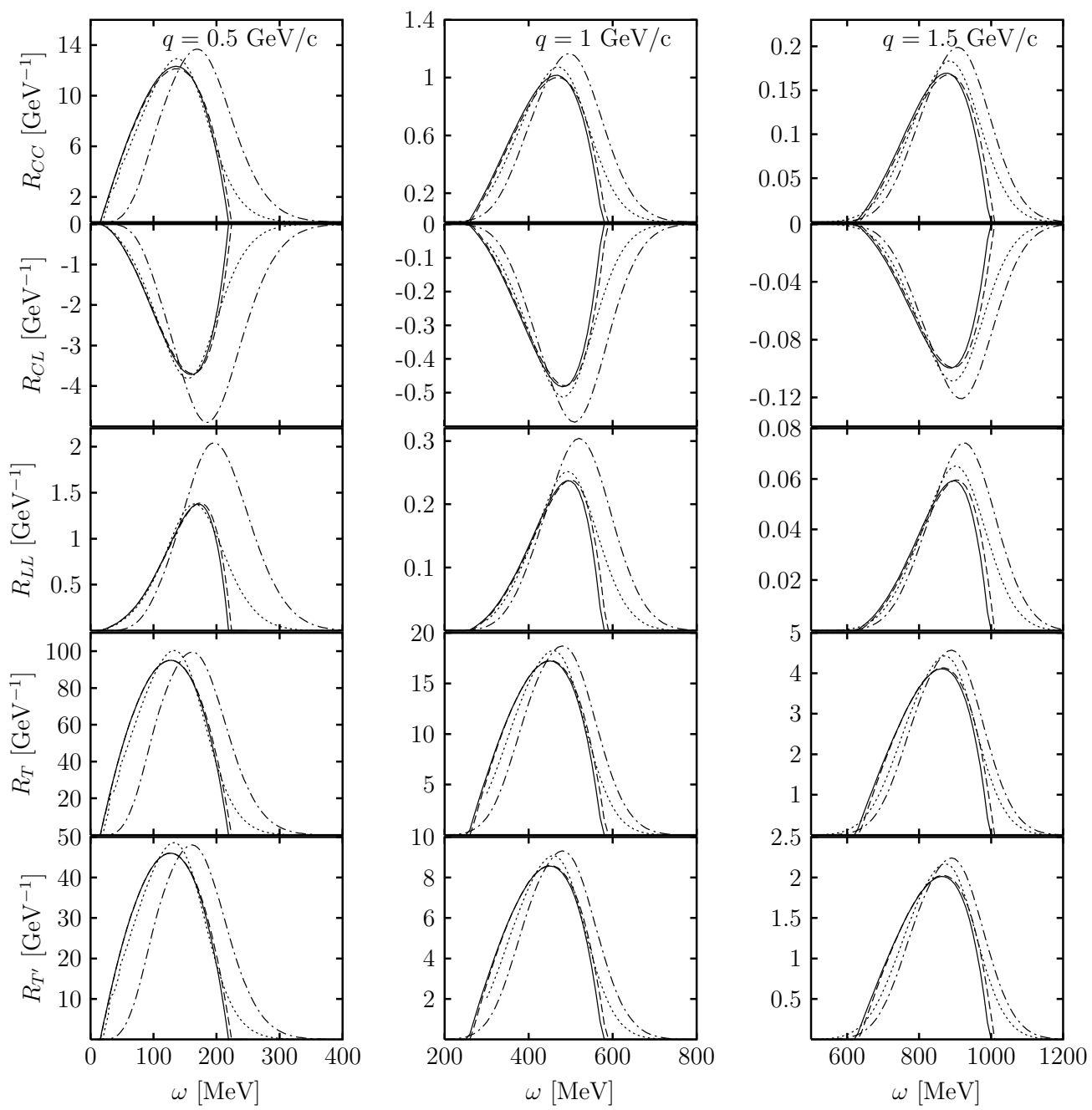

FIG. 3. Same as Fig. 1, but for the total (vector plus axial-vector) responses.

[see Eqs. (29) and (30)], is dominant over the zeroth-order contribution, and the corresponding response functions are in general small compared with the vector ones. An example is shown in Fig. 4, where we display the separate contributions of the zeroth- and first-order terms to the axial-vector response $R_{\mathrm{CC}}^{A}$ of ${ }^{12} \mathrm{C}$ for $q=500 \mathrm{MeV} / c$ in the CSM. For higher values of $q$ the zeroth-order contribution is much smaller than the others.

From inspection of the responses $R_{\mathrm{CC}}, R_{T}$, and $R_{T^{\prime}}$, we observe that the PWIA results are clearly shifted to the right of the CSM by roughly the averaged depth of the potential $\sim 35 \mathrm{MeV}$. This shift is present also in the separate vector and axial-vector responses. In the case of the $C L$ and $L L$, the shift is larger because of the energy factors used to compute the $L$ component of the current. The origin of the shift in PWIA is related to the different treatment of the nuclear Hamiltonian in the initial and final states [27]: while the energy of the initial bound neutron is the sum of kinetic plus potential, $\epsilon_{h}=t_{h}+v_{h}$, the exiting proton is a plane wave and has only kinetic energy $\epsilon_{p}=t_{p}$. Accordingly, for fixed $\omega$ there is an imbalance between potential energies in the initial and final states, yielding $\omega=t_{p}-t_{h}-v_{h}$. However, in the CSM we use the same potential for the initial and final state, which now has $\epsilon_{p}=t_{p}+v_{p}$. Hence $\omega=t_{p}-t_{h}+v_{p}-v_{h}$. Thus, in the CSM the potential energies of particle and hole partially cancel

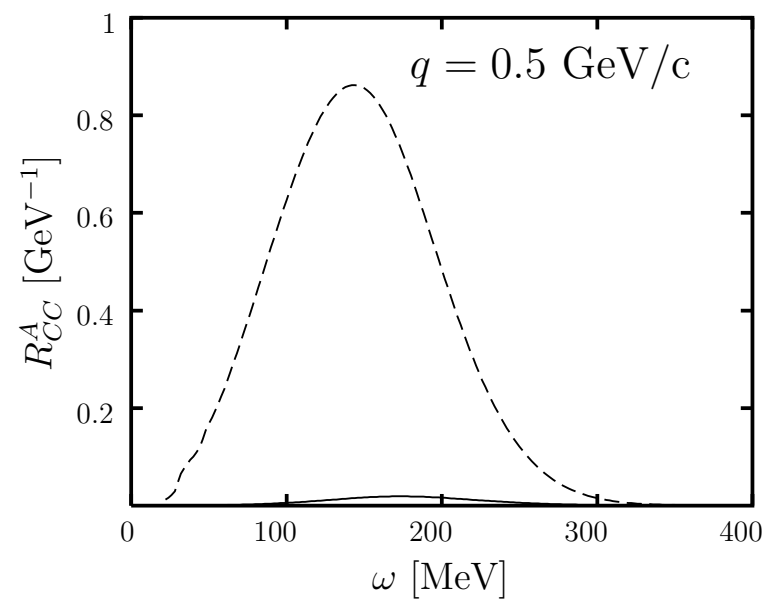

FIG. 4. Axial-vector response $R_{\mathrm{CC}}^{A}$ of ${ }^{12} \mathrm{C}$ in the CSM. Solid: contribution of the zeroth-order term in the SR expansion. Dashed: first-order contribution. 

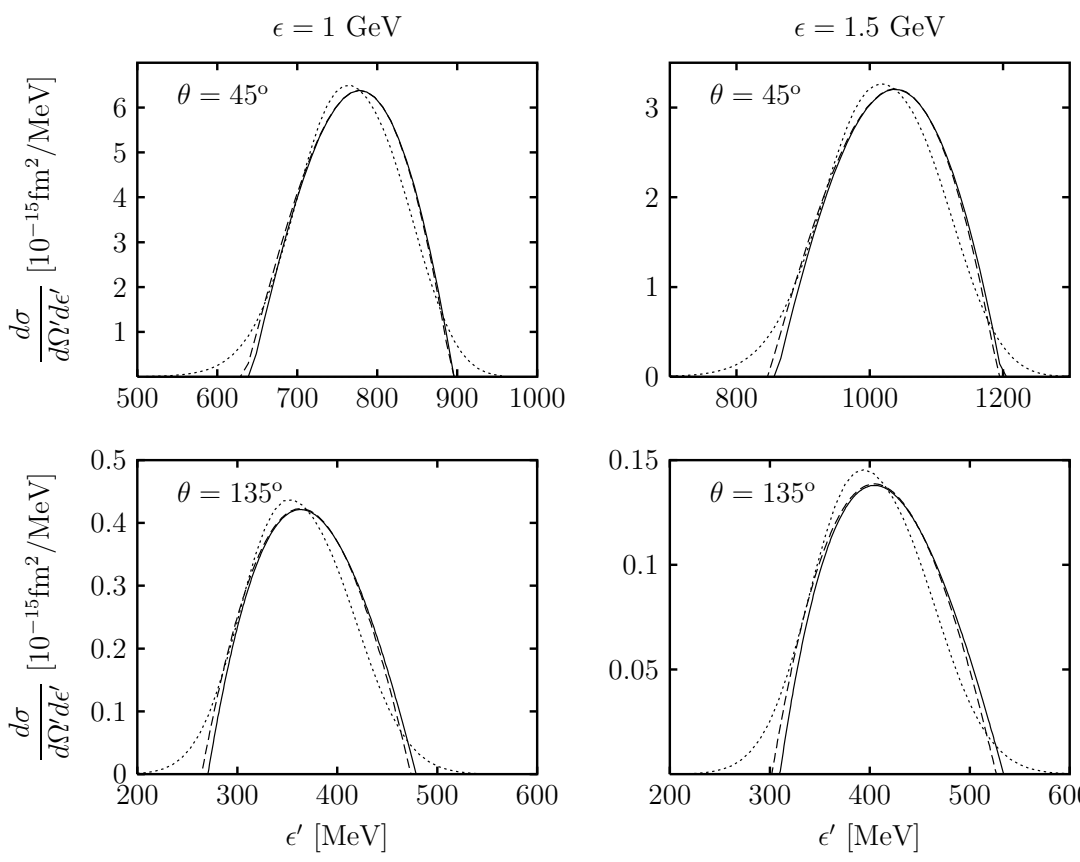

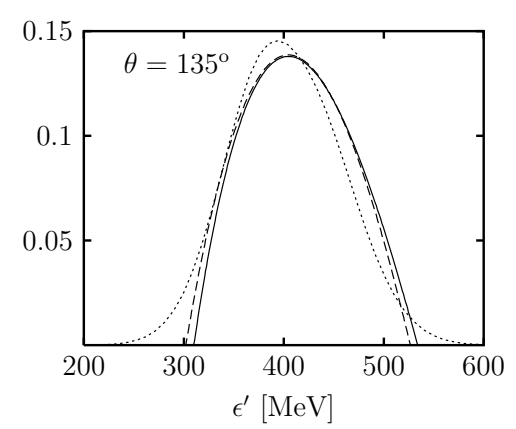

FIG. 5. Differential cross section of the reaction ${ }^{12} \mathrm{C}\left(v_{\mu}, \mu^{-}\right)$for incident neutrino energies $\epsilon=1$ and $1.5 \mathrm{GeV}$ and for two scattering angles. Solid: RFG with $k_{F}=215 \mathrm{MeV} / c$. Dashed: SRFG. Dotted: CSM. out; this explains why the position of the peak is close to the RFG, where only kinetic energies enter. Of course, the cancellation is not perfect, and a slight shift to the right of the RFG is also observed in the CSM. This small shift of the CSM is the behavior expected from previous theoretical studies [61].

Another test of the SR approximation is illustrated by the results shown in Fig. 5 for the differential cross section ${ }^{12} \mathrm{C}\left(v_{\mu}, \mu^{-}\right)$. Therein we show examples for two incident neutrino energies, $\epsilon=1$ and $1.5 \mathrm{GeV}$, and for two scattering angles $\theta=45^{\circ}$, and $135^{\circ}$, as a function of the exiting muon energy $\epsilon^{\prime}$; so in this case, we are testing different ranges of $q$ and $\omega$, which can be high or low depending on the kinematics. We see again that the RFG and SRFG predictions are almost equal in all cases, while the CSM also gives similar results, with the exception of the characteristic tails and slight shift, which now is to the left of the RFG because $\omega$ decreases with $\epsilon^{\prime}$.

Summarizing this subsection, using the Fermi gas as "testing arena" for the approximated CC current, we have found that the accord between the results obtained using the SR expansion and the exact relativistic result is almost perfect for the intermediate and high values of $q$ and $\omega$ considered. The CSM relativized using our procedure gives rise to cross sections that are within the allowed kinematical region and have a magnitude similar to those of the RFG. This behavior of the CSM is similar to what was found for $\left(e, e^{\prime}\right)$ reactions in [26]. We should underscore the fact that the CSM is used here just to illustrate how one can use our relativizing procedure in a more complex model than the Fermi gas and to show the results expected from it. In particular, especially when discussing lower energies where nonrelativistic approaches are valid, more elaborate models exist that describe the $\left(e, e^{\prime}\right)$ experimental data rather well. For example, it is well known from other work that final-state interactions can significantly modify the bare CSM or RPA responses, mainly through medium renormalization of the particle-hole excitations. This mechanism can be approximately taken into account by using an effective nucleon mass that produces a shift and by a folding of the bare responses, producing an asymmetric broadening which improves the agreement with the experimental data [62-64].

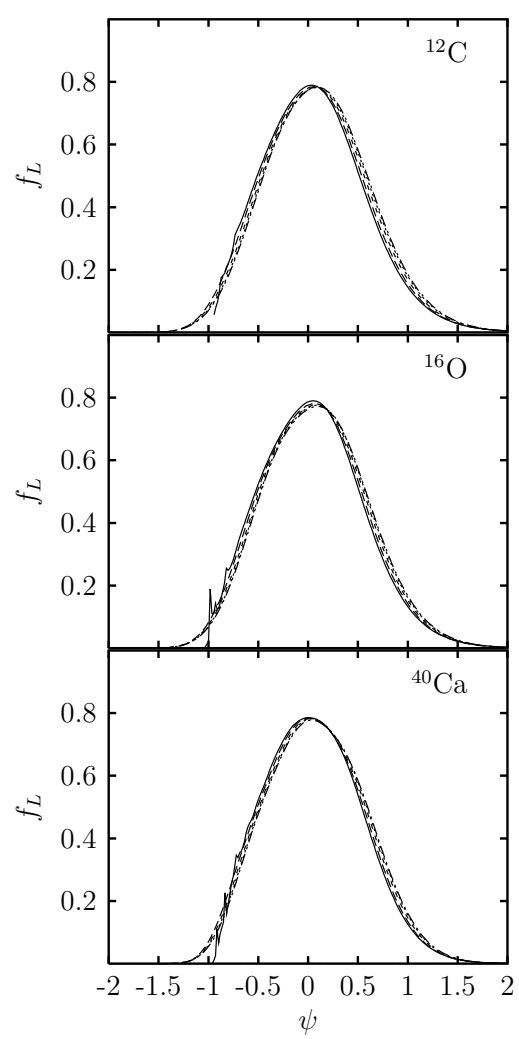

FIG. 6. Scaling of the first kind in the CSM with scaling functions obtained from the longitudinal electromagnetic responses in the CSM. In each panel we include $q=0.5,0.7,1,1.3$, and $1.5 \mathrm{GeV} / c$. 


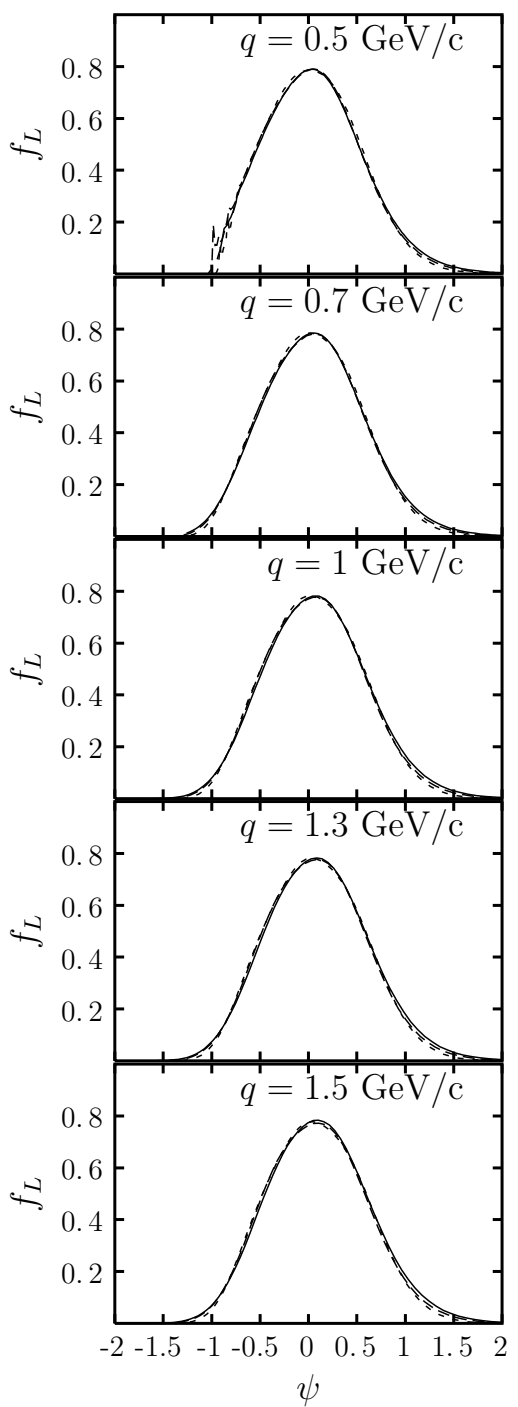

FIG. 7. Scaling of the second kind including in each panel the nuclei ${ }^{12} \mathrm{C},{ }^{16} \mathrm{O}$, and ${ }^{40} \mathrm{Ca}$.

\section{B. Scaling}

In this subsection we work within the CSM. We first focus on the inclusive electron scattering reaction and investigate the scaling properties of the electromagnetic responses for various choices of kinematics for ${ }^{12} \mathrm{C},{ }^{16} \mathrm{O}$, and ${ }^{40} \mathrm{Ca}$. The $\left(e, e^{\prime}\right)$ cross section is given by

$$
\frac{d \sigma}{d \Omega^{\prime} d \epsilon^{\prime}}=\sigma_{\mathrm{Mott}}\left(v_{L} R_{L}+v_{T} R_{T}\right) .
$$

The same SR expansion for the vector current, Eqs. (24) and (25), is employed here for the electromagnetic sector.

We compute the CSM scaling functions as

$$
\begin{aligned}
& f_{L}=\frac{R_{L}}{G_{L}}, \\
& f_{T}=\frac{R_{T}}{G_{T}},
\end{aligned}
$$
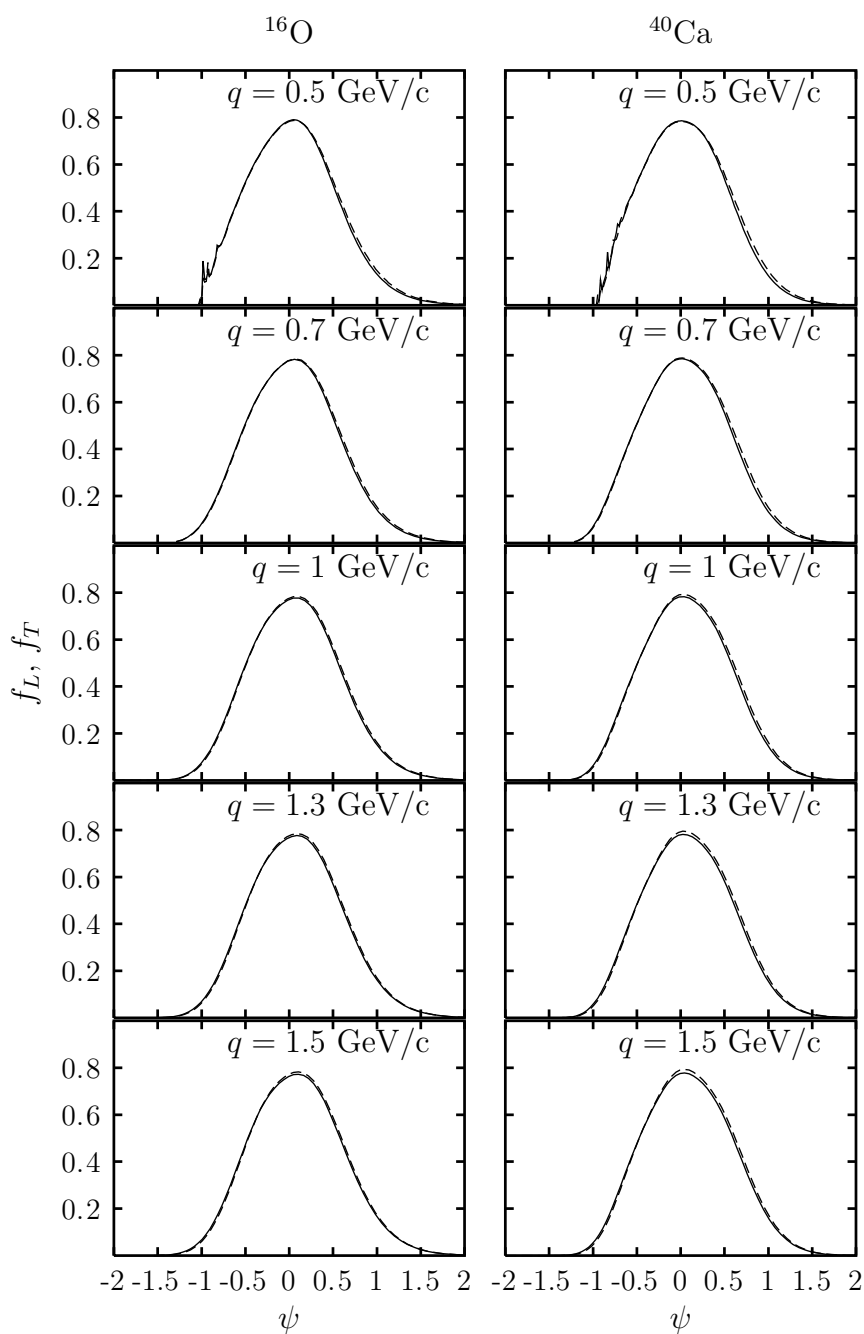

FIG. 8. Scaling function $f_{L}$ obtained from the longitudinal electromagnetic response (solid lines), compared with the scaling function $f_{T}$ obtained from the transverse response (dashed lines), for several values of $q$.

with

$$
G_{K}=\Lambda_{0}\left(Z U_{K}^{p}+N U_{K}^{n}\right) \quad K=L, T,
$$

where $\Lambda_{0}$ is given in Eq. (C2) in Appendix C, and the electromagnetic single-nucleon functions for protons $U_{K}^{p}$ and neutrons $U_{K}^{n}$ are defined similarly to the ones given in Appendix $\mathrm{C}$ for the vector current $U_{\mathrm{CC}}^{V}$ and $U_{T}^{V}$.

The scaling behavior can be studied by plotting these functions against the scaling variable [46]

$$
\psi=\frac{1}{\sqrt{\xi_{F}}} \frac{\lambda-\tau}{\sqrt{(1+\lambda) \tau+\kappa \sqrt{\tau(1+\tau)}}}
$$

for different kinematics and for different nuclei. Here $\xi_{F}=$ $\sqrt{1+k_{F}^{2} / m_{N}^{2}}-1$.

A study of the behavior of $f_{L}$, computed for various values of the momentum transfer, is summarized in Fig. 6. We plot together $f_{L}$ for $q=0.5,0.7,1,1.3$, and $1.5 \mathrm{GeV} / c$. All the curves approximately collapse into one. Small violations of the scaling are seen at low $\psi$ coming from the low-energy 

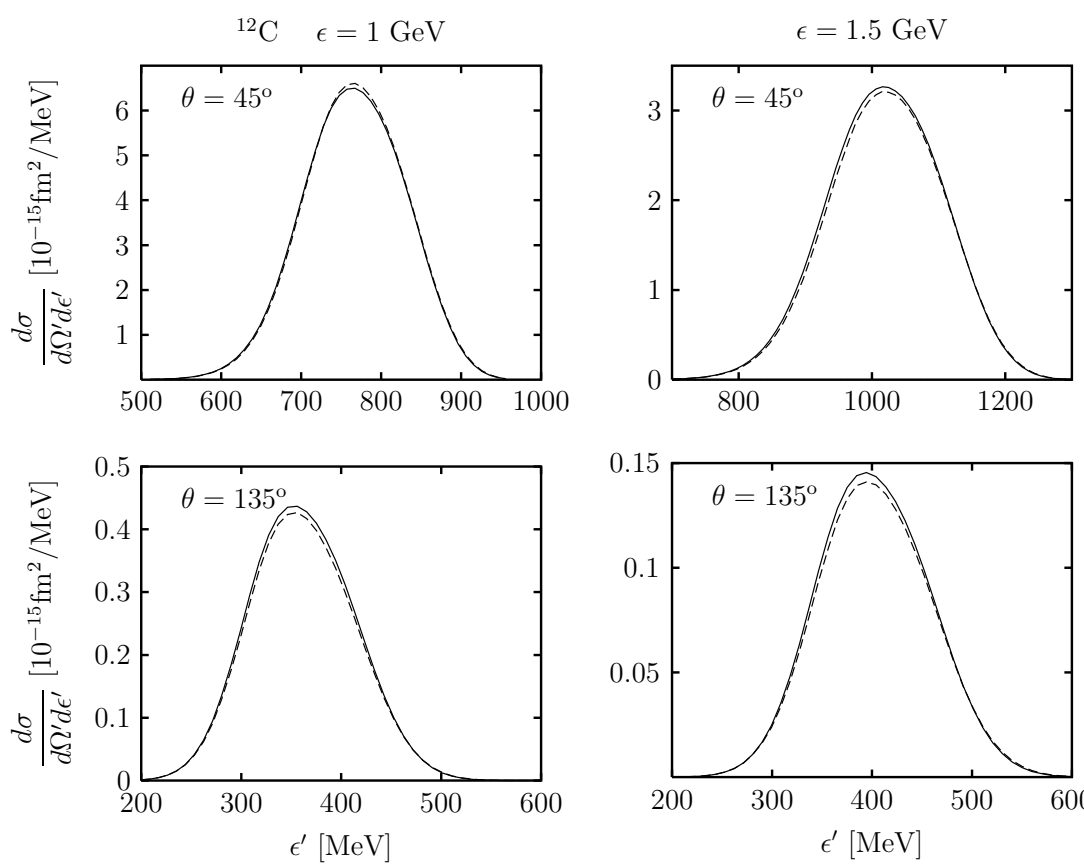

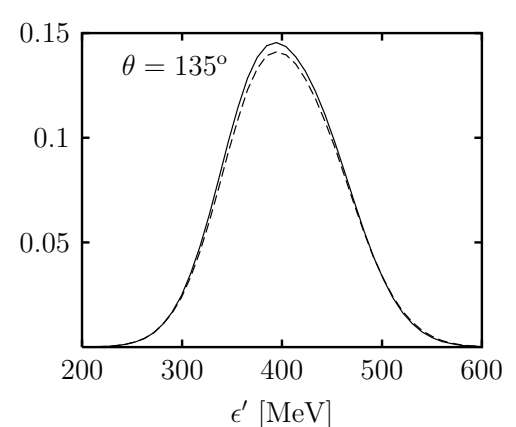

FIG. 9. Differential cross section of the reaction ${ }^{12} \mathrm{C}\left(v_{\mu}, \mu^{-}\right)$for neutrino incident energies $\epsilon=1$ and $1.5 \mathrm{GeV}$ and for two scattering angles. Solid: CSM. Dashed: reconstructed from the electromagnetic scaling function $f_{L}(\psi)$ computed at the same kinematics. potential resonances for $q=0.5 \mathrm{GeV} / c$, which disappear for higher $q$ values. Thus, scaling of the first kind is approximately achieved in the CSM. Note that scaling also holds for $|\psi|>1$, the region where the RFG responses are zero.

Scaling of the second kind, i.e., independence of the nuclear species for fixed $q$, is illustrated in Fig. 7. We plot together $f_{L}$ for the three nuclei studied. The fitted Fermi momenta are 220, 215 , and $240 \mathrm{MeV} / \mathrm{c}$ for ${ }^{12} \mathrm{C},{ }^{16} \mathrm{O}$, and ${ }^{40} \mathrm{Ca}$, respectively. The collapse of the three curves into one is clearly seen, with small deviations at the region of the maximum. Again the exception is found for $q=0.5 \mathrm{GeV} / c$ in the resonance region. Since both kinds of scaling are found, we conclude that superscaling occurs within our model.
Finally, in Fig. 8 we show what has been called scaling of the zeroth kind [50], i.e., the longitudinal and transverse scaling functions, $f_{L}$ and $f_{T}$, also collapse into one universal function $f$. Experimentally, deviations from scaling in the region of the QE peak mainly occur in the transverse response and are related to contributions beyond the impulse approximation, in particular to meson-exchange currents [49,50]. Since these contributions are not included in our CSM, the $L$ and $T$ responses scale in the same way, as shown in Fig. 8.

In the present work, we do not compare with the experimental $\left(e, e^{\prime}\right)$ scaling function because, as stated above, the CSM is still lacking some ingredients (for instance, medium modifications to the $\mathrm{p}$-h propagator, inclusion of
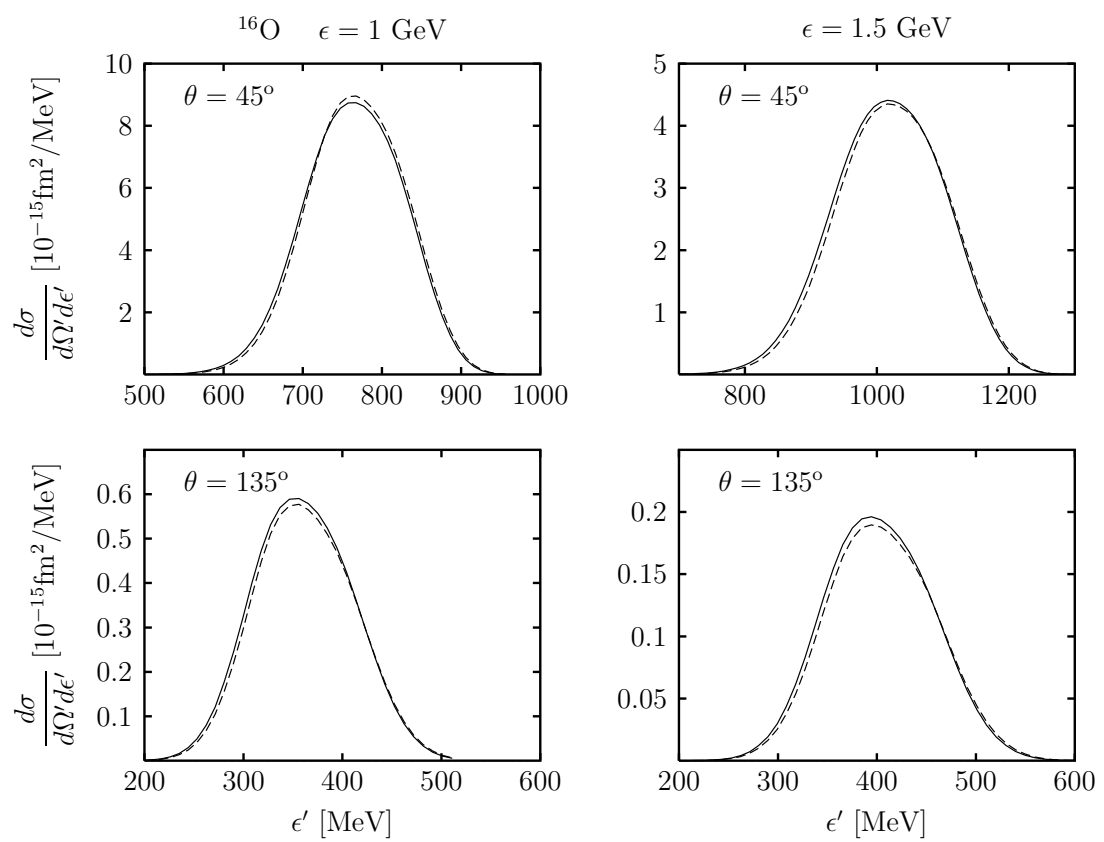

FIG. 10. Same as Fig. 9, but for ${ }^{16} \mathrm{O}$. 
energy-dependent potentials, etc.), without which one should not expect to obtain excellent agreement with the experimental data. The focus of the present study has been limited to showing that superscaling occurs at the level of relativized CSM.

Let us now turn to neutrino reactions. In [53] a semiempirical model, based on the superscaling property of the $L$ responses of ${ }^{12} \mathrm{C}$ and ${ }^{16} \mathrm{O}$ for high momentum transfer, was proposed to predict the neutrino inclusive cross sections up to the $\Delta$ peak. The latter were calculated starting from the RFG expression for the cross sections and substituting the "theoretical" RFG superscaling functions with phenomenological ones (one for the QE and a different one for the $\Delta$ peak) derived from fits of electron scattering data. Therefore, this reconstruction, besides assuming the validity of superscaling in electron scattering, relies on the hypothesis that it also holds for the neutrino inclusive cross section for the high energies involved, so that electron scattering results can safely be used as input in neutrino scattering calculations. This assumption obviously cannot be tested using the RFG model, since, as stated in the introduction, it is true by construction.

In the present work, we have at hand a model, the CSM, for both the QE $\left(e, e^{\prime}\right)$ and $\left(v_{\mu}, \mu^{-}\right)$reactions that has been relativized and so should be able to handle modeling at high energies. Moreover, superscaling of the electromagnetic responses is well satisfied by the CSM. Hence we can adopt the same approach as in [53] treating the CSM electromagnetic scaling function as a pseudophenomenological one and using it to compute neutrino cross sections. Upon comparing cross sections obtained this way with those obtained directly using the CSM, we can gain some insight into the degree to that the shell model incorporates effects that are scale breaking. While the level of scaling violation is expected to be quite small, since we have already seen excellent superscaling in the figures discussed above, the specific differences in the roles played by the various current operators involved in electromagnetic and $\mathrm{CC}$ weak processes might lead to different sensitivities to scale-breaking effects. Accordingly, it is useful to compare the cross sections obtained within the scaling approach with those computed directly using the model. In context, it should be noted that this does not imply in general that the scaling approach has been shown to be robust, since here the comparisons are being made entirely within limited models which clearly lack elements that may play some role in the responses and may be scale breaking. We know of at least one such ingredient, namely, meson-exchange currents which have been shown to provide scale-breaking corrections that enter typically at the $10 \%$ level in the overall cross sections. Nevertheless, the study presented below indicates that the scale-breaking effects incorporated in the present models are in fact quite small.

We show typical results of this study in Figs. 9 and 10 for ${ }^{12} \mathrm{C}$ and ${ }^{16} \mathrm{O}$, respectively. The cross section for $\left(v_{\mu}, \mu^{-}\right)$is shown for two incident energies and for two scattering angles. The dashed lines have been computed from the scaling function $f_{L}$ obtained from the analysis of $\left(e, e^{\prime}\right)$ CSM predictions. To be precise, we use Eq. (C1) to compute the neutrino responses, substituting $f^{\mathrm{RFG}}$ by $f_{L}$. The solid lines correspond to the exact CSM result. The differences found between the two approaches are quite small, at most $\sim 3 \%$. These small differences are produced by the slightly different scaling behavior of the axial-vector and vector responses, which, however, has little effect on the total cross section. Due to the simplicity of the CSM, these results cannot be taken as a definite proof of superscaling, but they certainly represent an important step forward in establishing the validity of the approach presented in [53].

\section{CONCLUSIONS}

One of the goals of the present study was to explore the degree to which scaling and superscaling behaviors are reached for relatively high-energy semileptonic inclusive reactions with nuclei at excitation energies in the vicinity of the quasielastic peak. The focus has been placed on comparisons between the Fermi gas model (both the fully relativistic Fermi gas and a semirelativized version of it) and a relativized continuum shell model.

Since we are interested in energies of several GeV, relativity is clearly a required ingredient. In the present work, we have presented a clear and direct procedure that allows one to incorporate some classes of relativistic effects and thereby to relativize otherwise nonrelativistic models of the reaction, such as the shell model employed here. We use the two versions of the Fermi gas model to motivate the procedures followed. Two steps are involved: first, one relativizes the kinematics in the reaction, and second, expansions are made for the single-nucleon currents in the problem. In this work, we have extended our previous treatments to include the full charge-changing weak interaction current. Importantly, because the expansions are made to first order in $p / m_{N}$, but not at all in $q / m_{N}$ or $\omega / m_{N}$, they differ from the traditional FW expansions, and for QE scattering they should be more robust at high energies where expansions in $q / m_{N}$ and $\omega / m_{N}$ clearly fail.

Using the two versions of the Fermi gas model and the relativized continuum shell model, together with some results from the plane-wave impulse approximation, to represent both electromagnetic $\left(e, e^{\prime}\right)$ and $\mathrm{CC}$ weak $\left(v_{\mu}, \mu^{-}\right)$processes, we have proceeded to quantify the level of scaling violation. This allows us to evaluate the corresponding uncertainty in the predictions one makes for the neutrino cross section using the scaling approach with input from analyses of electron scattering data. In the present paper, we have shown that for intermediate to high energies the description of the final state through a real mean field seems not to change appreciably the scaling and superscaling properties of the electromagnetic response functions at the quasielastic peak, and that the scaling function extracted from these is basically the same as for the $\left(v_{\mu}, \mu^{-}\right)$reaction.

Although the present analysis performed within the relativized shell model does not constitute a proof of the scaling hypothesis in the general case, scaling studies such as those presented here allow us to gain additional insight and, in particular, to rule out (or not) some reaction mechanisms as possible causes of scaling violations.

In summary, the relativizing procedure followed in this work embodies some of the ingredients that are almost 
certainly required for a successful description of such inclusive processes in the quasielastic regime. Models that do not take at least these ingredients into account are very likely to fail in the several-GeV energy region of interest here. This does not mean, however, that additional dynamical features are not needed before a full understanding of these high-energy responses will be attained. In particular, other parallel studies being pursued by us hold some promise for reaching a good understanding of the phenomenologically derived scaling function used recently to make predictions for CC neutrino reactions in the $\mathrm{GeV}$ region. Some of these results will be presented in the near future.

\section{ACKNOWLEDGMENTS}

This work was partially supported by funds provided by DGI (Spain) and FEDER funds, under Contract Nos. BFM2002-03218, BFM2002-03315, and FPA200204181-C04-04. and by the Junta de Andalucía and the INFN-CICYT Collaboration agreement (Project "Study of Relativistic Dynamics in Electron and Neutrino Scattering"). It was also supported in part (TWD) by the U.S. Department of Energy under cooperative research agreement No. DE-FC0294ER40818.

\section{APPENDIX A: MULTIPOLES OF THE CONVECTIVE AXIAL-VECTOR OPERATOR}

Here we compute the Coulomb multipoles of the convective axial-vector charge operator, which is the first-order term in the expansion of $j_{A}^{0}$ [Eq. (29)]. The corresponding coordinatespace operator is given by

$$
\rho_{C}(\mathbf{q}) \equiv e^{i \mathbf{q} \cdot \mathbf{r}} \zeta_{0}^{\prime \prime} \boldsymbol{\eta}_{\perp} \cdot \boldsymbol{\sigma},
$$

where $\mathbf{r}$ is the coordinate of the nucleon over which the operator acts. To obtain the multipole operators, we write the above operator in the form

$$
\begin{aligned}
\rho_{C}(\mathbf{q}) & =e^{i \mathbf{q} \cdot \mathbf{r}} \zeta_{0}^{\prime \prime}\left(\boldsymbol{\eta}-\frac{\boldsymbol{\eta} \cdot \boldsymbol{q}}{q^{2}} \mathbf{q}\right) \cdot \boldsymbol{\sigma} \\
& =\zeta_{0}^{\prime \prime} \boldsymbol{\sigma} \cdot\left(\boldsymbol{\eta}+\frac{\boldsymbol{\eta} \cdot \nabla}{q^{2}} \nabla\right) e^{i \mathbf{q} \cdot \mathbf{r}} .
\end{aligned}
$$

Performing in this equation the multipole expansion of the plane wave for $\mathbf{q}$ along the $z$ axis

$$
e^{i \mathbf{q} \cdot \mathbf{r}}=\sqrt{4 \pi} \sum_{J} i^{J}[J] j_{J}(q r) Y_{J 0}(\hat{\mathbf{r}}),
$$

where $[J]=\sqrt{2 J+1}$, we obtain

$$
\rho_{C}(\mathbf{q})=\sqrt{4 \pi} \sum_{J} i^{J}[J] \hat{C}_{J 0}(q),
$$

where the Coulomb multipole operators are

$$
\hat{C}_{J 0}(q)=\zeta_{0}^{\prime \prime} \sigma \cdot\left(\eta+\frac{\eta \cdot \nabla}{q^{2}} \nabla\right) j_{J}(q r) Y_{J 0}(\hat{\mathbf{r}}) .
$$

Later on we will also make the substitution $\mathbf{p} \rightarrow-i \nabla$, although in this equation $\nabla$ operates only on $j_{J}(q r) Y_{J 0}(\hat{\mathbf{r}})$.
The procedure now is to use Racah algebra and the general properties of spherical harmonics and spherical Bessel functions to write the above operator in explicit sphericaltensor form. The following expressions can be obtained:

$$
\begin{aligned}
\hat{C}_{J 0} & =\zeta_{0}^{\prime \prime}\left[\hat{C}_{J 0}^{(1)}+\hat{C}_{J 0}^{(2)}\right], \\
\hat{C}_{J 0}^{(1)} & =\frac{i}{m} \frac{1}{[J]} \sum_{L}[L] U_{J L J}, \\
\hat{C}_{J 0}^{(2)} & =-\frac{i}{m} \sum_{s= \pm 1} \sum_{s^{\prime}= \pm 1} \frac{\left(J+\delta_{s 1}\right)^{1 / 2}\left(J^{\prime}+\delta_{s^{\prime} 1}\right)^{1 / 2}}{[J]\left[J^{\prime}\right]} U_{J^{\prime \prime} J^{\prime} J},
\end{aligned}
$$

where $J^{\prime}=J+s, J^{\prime \prime}=J^{\prime}+s^{\prime}$, and $s, s^{\prime}= \pm 1$ (coming from the derivative of the Bessel function, which is a linear combination of the Bessel functions for $J \pm 1$ ), and we have defined the auxiliary coupled operator

$$
U_{J^{\prime \prime} J^{\prime} J} \equiv j_{J^{\prime \prime}}(q r)\left[\sigma \otimes\left[Y_{J^{\prime \prime}}(\hat{\mathbf{r}}) \otimes \nabla\right]_{J^{\prime}}\right]_{J 0} .
$$

Next we proceed to compute the reduced matrix elements of this $U$ operator between shell model particle and hole states $|p\rangle=\left|\frac{1}{2} l_{p} j_{p}\right\rangle$ and $|h\rangle=\left|\frac{1}{2} l_{h} j_{h}\right\rangle$. Using standard Racah algebra $[65,66]$ we obtain

$$
\begin{aligned}
& \left\langle p\left\|U_{J^{\prime \prime} J^{\prime} J}\right\| h\right\rangle \\
& =(-)^{l_{h}+J^{\prime}} \sqrt{\frac{3}{2 \pi}}\left[l_{p}\right]\left[j_{p}\right]\left[j_{h}\right][J]\left[J^{\prime}\right]\left[J^{\prime \prime}\right]\left\{\begin{array}{ccc}
\frac{1}{2} & l_{p} & j_{p} \\
\frac{1}{2} & l_{h} & j_{h} \\
1 & J^{\prime} & J
\end{array}\right\} \\
& \quad \times \sum_{s_{h}= \pm 1}\left[L_{h}\right] s_{h}\left(l_{h}+\delta_{s_{h}}\right)^{1 / 2}\left\{\begin{array}{lll}
J^{\prime \prime} & 1 & J^{\prime} \\
l_{h} & l_{p} & L_{h}
\end{array}\right\}\left(\begin{array}{ccc}
l_{p} & J^{\prime \prime} & L_{h} \\
0 & 0 & 0
\end{array}\right) \\
& \quad \times \int_{0}^{\infty} d r r^{2} R_{p}(r) j_{J^{\prime \prime}}(q r)\left(\frac{d}{d r}-s_{h} \frac{l_{h}+\delta_{s_{h},-1}}{r}\right) R_{h}(r),
\end{aligned}
$$

where $R_{p}(r)$ and $R_{h}(r)$ are the radial-wave functions, $L_{h}=$ $l_{h}+s_{h}$, and $s_{h}= \pm 1$. We can now use this result in Eqs. (A8) and (A9). It is convenient to use the following identities involving products of six- $j$ and three- $j$ coefficients, for $J^{\prime \prime}=$ $J^{\prime}+s^{\prime}$ and $L_{h}=l_{h}+s_{h}$, with $s^{\prime}, s_{h}= \pm 1$ :

$$
\begin{aligned}
& \left\{\begin{array}{lll}
J^{\prime \prime} & 1 & J^{\prime} \\
l_{h} & l_{p} & L_{h}
\end{array}\right\}\left(\begin{array}{ccc}
l_{p} & J^{\prime \prime} & L_{h} \\
0 & 0 & 0
\end{array}\right) \\
& =\frac{P_{l_{p}+l_{h}+J^{\prime}}^{+}}{\left[L_{h}\right]\left[l_{h}\right]\left[J^{\prime}\right]\left[J^{\prime \prime}\right]}\left\{\left[\left(l_{h}+\delta_{s_{h},-1}\right)\left(J^{\prime}+\delta_{s^{\prime},-1}\right)\right]^{1 / 2}\right. \\
& \times\left(\begin{array}{ccc}
l_{h} & J^{\prime} & l_{p} \\
1 & -1 & 0
\end{array}\right)-s_{h} s^{\prime}\left[\left(l_{h}+\delta_{s_{h}, 1}\right)\left(J^{\prime}+\delta_{s^{\prime}, 1}\right)\right]^{1 / 2} \\
& \left.\times\left(\begin{array}{ccc}
l_{h} & J^{\prime} & l_{p} \\
0 & 0 & 0
\end{array}\right)\right\} \\
& \left\{\begin{array}{ccc}
J & 1 & J \\
l_{h} & l_{p} & L_{h}
\end{array}\right\}\left(\begin{array}{ccc}
l_{p} & J & L_{h} \\
0 & 0 & 0
\end{array}\right) \\
& =\frac{P_{l_{p}+l_{h}+J+1}^{+}}{\left[L_{h}\right]\left[l_{h}\right][J]}\left(l_{h}+\delta_{s_{h},-1}\right)\left(\begin{array}{ccc}
l_{h} & J & l_{p} \\
1 & -1 & 0
\end{array}\right),
\end{aligned}
$$

where $P_{n}^{+}$is the parity function equal to 1 if $n$ is even and zero if $n$ is odd. We also use the following product of a nine- $j$ and 
a three- $j$

$$
\begin{aligned}
\left\{\begin{array}{ccc}
\frac{1}{2} & l_{p} & j_{p} \\
\frac{1}{2} & l_{h} & j_{h} \\
1 & J^{\prime} & J
\end{array}\right\}\left(\begin{array}{ccc}
l_{h} & J^{\prime} & l_{p} \\
0 & 0 & 0
\end{array}\right) \\
=\frac{(-)^{j_{p}+l_{p}+\frac{1}{2}}}{\sqrt{6}} \frac{P_{l_{p}+l_{h}+J+1}^{+}}{\left[l_{p}\right]\left[l_{h}\right][J]\left[J^{\prime}\right]} \\
\quad \times \frac{\chi_{p}+\chi_{h}+s J+\delta_{s 1}}{\sqrt{J+\delta_{s 1}}}\left(\begin{array}{ccc}
j_{p} & j_{h} & J \\
\frac{1}{2} & -\frac{1}{2} & 0
\end{array}\right),
\end{aligned}
$$

where $\chi_{p}=(-1)^{l_{p}+j_{p}+\frac{1}{2}}\left(j_{p}+\frac{1}{2}\right)$. After some work, we finally arrive at the matrix elements written in the form

$$
\begin{aligned}
\left\langle p\left\|\hat{C}_{J}^{(1)}\right\| h\right\rangle= & \frac{i}{m} A_{J}(p h) \int_{0}^{\infty} d r r R_{p}^{*}(r) j_{J}(q r) R_{h}(r) \\
& +\frac{i}{m} B_{J}(p h) \int_{0}^{\infty} d r r^{2} R_{p}^{*}(r) j_{J}(q r) \frac{d R_{h}(r)}{d r},
\end{aligned}
$$

$$
\begin{aligned}
\left\langle p\left\|\hat{C}_{J}^{(2)}\right\| h\right\rangle= & \frac{i}{m} \sum_{s= \pm 1} A_{J J^{\prime}}(p h) \frac{1}{q} \int_{0}^{\infty} d r R_{p}^{*}(r) j_{J^{\prime}}(q r) R_{h}(r) \\
& +\frac{i}{m} \sum_{s= \pm 1} B_{J J^{\prime}}(p h) \int_{0}^{\infty} d r r^{2} R_{p}^{*}(r) j_{J^{\prime}}^{\prime}(q r) \frac{d R_{h}(r)}{d r}
\end{aligned}
$$

where $J^{\prime}=J+s, s= \pm 1$, and $j_{J^{\prime}}^{\prime}(z)$ is the derivative of the Bessel function. We have defined the following coefficients,

$$
\begin{aligned}
& A_{J}(p h)=P_{l_{p}+l_{h}+J+1}^{+}\left[[J] \sqrt{l_{h}\left(l_{h}+1\right)} b_{J J}\right. \\
& \left.-\sum_{s= \pm 1} \frac{\left[J^{\prime}\right]}{[J]} \sqrt{J^{\prime}+\delta_{s 1}} b_{J J^{\prime}}\right] a_{J}, \\
& B_{J}(p h)=P_{l_{p}+l_{h}+J+1}^{+} \frac{(-)^{j_{p}+\frac{1}{2}}}{\sqrt{4 \pi}}\left[j_{p}\right]\left[j_{h}\right][J]\left(\begin{array}{ccc}
j_{p} & j_{h} & J \\
\frac{1}{2} & -\frac{1}{2} & 0
\end{array}\right) a_{J}, \\
& A_{J J^{\prime}}(p h) \\
& =P_{l_{p}+l_{h}+J+1}^{+} \sqrt{J+\delta_{s 1}} \sqrt{l_{h}\left(l_{h}+1\right)} \sqrt{J^{\prime}\left(J^{\prime}+1\right)} a_{J^{\prime}} b_{J J^{\prime}},
\end{aligned}
$$

$$
\begin{aligned}
& B_{J J^{\prime}}(p h) \\
& =P_{l_{p}+l_{h}+J+1}^{+} \frac{(-)^{j_{p}-1 / 2}}{\sqrt{4 \pi}} \frac{\left[j_{p}\right]\left[j_{h}\right]}{[J]}\left(\chi_{p}+\chi_{h}+s J+\delta_{s 1}\right) \\
& \quad \times\left(\begin{array}{ccc}
j_{p} & j_{h} & J \\
\frac{1}{2} & -\frac{1}{2} & 0
\end{array}\right)
\end{aligned}
$$

where the factors $a_{J}$ and $b_{J J^{\prime}}$ are defined as

$$
\begin{aligned}
a_{J} & =(-)^{l_{p}} \sqrt{\frac{3}{2 \pi}}\left[l_{p}\right]\left[l_{h}\right]\left[j_{p}\right]\left[j_{h}\right][J], \\
b_{J J^{\prime}} & =\left\{\begin{array}{lll}
\frac{1}{2} & l_{p} & j_{p} \\
\frac{1}{2} & l_{h} & j_{h} \\
1 & J^{\prime} & J
\end{array}\right\}\left(\begin{array}{ccc}
l_{h} & J^{\prime} & l_{p} \\
1 & -1 & 0
\end{array}\right) .
\end{aligned}
$$

\section{APPENDIX B: PLANE WAVE IMPULSE APPROXIMATION}

We follow the approach of [27]. In PWIA, the inclusive response functions of the shell $h$ can be written as an integral over the missing momentum $\mathbf{p}=\mathbf{p}^{\prime}-\mathbf{q}$, with $p^{\prime}=\sqrt{2 m_{N} \epsilon_{p}}$,

$$
\left[R_{K}^{\mathrm{PWIA}}(q, \omega)\right]_{h}=\frac{m_{N}}{q} \int_{\left|p^{\prime}-q\right|}^{p+q} d p p \int_{0}^{2 \pi} d \phi w_{K}\left(\mathbf{p}^{\prime}, \mathbf{p}\right) M_{h}(\mathbf{p})
$$

of the scalar momentum distribution for each occupied shell

$$
M_{h}(\mathbf{p})=\frac{2 j_{h}+1}{4 \pi}\left|\tilde{R}_{h}(p)\right|^{2},
$$

where $\tilde{R}_{h}(p)$ is the radial-wave function in momentum space. The single-nucleon exclusive responses $w_{K}\left(\mathbf{p}^{\prime}, \mathbf{p}\right)$, for $K=$ CC, CL, LL, $T, T^{\prime}$ are readily computed using the vector, Eqs. (24)-(25), and axial-vector, Eqs. (28)-(30), current components

$$
\begin{aligned}
w_{\mathrm{CC}} & =w_{\mathrm{CC}}^{V}+w_{\mathrm{CC}}^{A}, \\
w_{\mathrm{CC}}^{V} & =\xi_{0}^{2}+\xi_{0}^{\prime 2} \kappa^{2} \eta_{\perp}^{2}, \\
w_{\mathrm{CC}}^{A} & =\zeta_{0}^{\prime 2} \kappa^{2}+\zeta_{0}^{\prime \prime 2} \eta_{\perp}^{2}, \\
w_{\mathrm{CL}} & =w_{\mathrm{CL}}^{V}+w_{\mathrm{CL}}^{A}, \\
w_{\mathrm{CL}}^{V} & =-\frac{\lambda}{\kappa} w_{\mathrm{CC}}^{V}, \\
w_{\mathrm{CL}}^{A} & =-\zeta_{0}^{\prime} \zeta_{3}^{\prime} \kappa^{2}-\zeta_{0}^{\prime \prime} \zeta_{3}^{\prime \prime} \eta_{\perp}^{2}, \\
w_{\mathrm{LL}} & =w_{\mathrm{LL}}^{V}+w_{\mathrm{LL}}^{A}, \\
w_{\mathrm{LL}}^{V} & =\left(\frac{\lambda}{\kappa}\right)^{2} w_{\mathrm{CC}}^{V}, \\
w_{\mathrm{LL}}^{A} & =\zeta_{3}^{\prime 2} \kappa^{2}+\zeta_{3}^{\prime 2} \eta_{\perp}^{2}, \\
w_{T} & =w_{T}^{V}+w_{T}^{A}, \\
w_{T}^{V} & =2 \xi_{1}^{\prime 2} \kappa^{2}+\xi_{1}^{2} \eta_{\perp}^{2}, \\
w_{T}^{A} & =2 \zeta_{1}^{\prime 2}, \\
w_{T^{\prime}} & =2 \xi_{1}^{\prime} \zeta_{1}^{\prime} \kappa .
\end{aligned}
$$

Since these functions do not depend on the azimuthal angle of $\mathbf{p}$, namely, $\phi$, the response functions in Eq. (B1) are reduced to an integral over the missing momentum $p$, which is performed numerically.

\section{APPENDIX C: THE RELATIVISTIC FERMI GAS}

Here we summarize the expressions for $\left(v_{\mu}, \mu^{-}\right)$reactions in the RFG model. We follow [53], where the expressions were 
written to leading order. Here we write the full results for the non-Pauli blocked regime of interest in this work. The nuclear response functions are written as

$$
R_{K}=N \Lambda_{0} U_{K} f_{\mathrm{RFG}}(\psi), \quad K=C C, C L, L L, T, T^{\prime},
$$

where $N$ is the neutron number, and

$$
\Lambda_{0}=\frac{\xi_{F}}{m_{N} \eta_{F}^{3} \kappa} .
$$

Here $\eta_{F}=k_{F} / m_{N}$, and $\xi_{F}=\sqrt{1+\eta_{F}^{2}}-1$. In Eq. (C1) $f_{\mathrm{RFG}}(\psi)$ is the scaling function of the RFG,

$$
f_{\mathrm{RFG}}(\psi)=\frac{3}{4}\left(1-\psi^{2}\right) \theta\left(1-\psi^{2}\right),
$$

and $\psi$ is the scaling variable given in Eq. (55). Finally, we give the single-nucleon responses $U_{K}$. For $K=C C$ we have

$$
\begin{aligned}
& U_{\mathrm{CC}}=U_{\mathrm{CC}}^{V}+\left(U_{\mathrm{CC}}^{A}\right)_{\mathrm{c} .}+\left(U_{\mathrm{CC}}^{A}\right)_{\text {n.c. }}, \\
& U_{\mathrm{CC}}^{V}=\frac{\kappa^{2}}{\tau}\left[\left(2 G_{E}^{V}\right)^{2}+\frac{\left(2 G_{E}^{V}\right)^{2}+\tau\left(2 G_{M}^{V}\right)^{2}}{1+\tau} \Delta\right],
\end{aligned}
$$

where

$$
\Delta=\frac{\tau}{\kappa^{2}} \xi_{F}\left(1-\psi^{2}\right)\left[\kappa \sqrt{1+\frac{1}{\tau}}+\frac{\xi_{F}}{3}\left(1-\psi^{2}\right)\right],
$$

and we have written the axial-vector response as the sum of conserved (c.) plus nonconserved (n.c.) parts,

$$
\begin{gathered}
\left(U_{\mathrm{CC}}^{A}\right)_{\text {c. }}=\frac{\kappa^{2}}{\tau} G_{A}^{2} \Delta, \\
\left(U_{\mathrm{CC}}^{A}\right)_{\text {n.c. }}=\frac{\lambda^{2}}{\tau} G_{A}^{\prime}{ }^{2} .
\end{gathered}
$$

For $K=C L, L L$ we have

$$
\begin{aligned}
& U_{\mathrm{CL}}=U_{\mathrm{CL}}^{V}+\left(U_{\mathrm{CL}}^{A}\right)_{\text {c. }}+\left(U_{\mathrm{CL}}^{A}\right)_{\text {n.c. }}, \\
& U_{\mathrm{LL}}=U_{\mathrm{LL}}^{V}+\left(U_{\mathrm{LL}}^{A}\right)_{\text {c. }}+\left(U_{\mathrm{LL}}^{A}\right)_{\text {n.c. }},
\end{aligned}
$$

where the vector and conserved axial-vector parts are determined by current conservation as

$$
\begin{aligned}
U_{\mathrm{CL}}^{V} & =-\frac{\lambda}{\kappa} U_{\mathrm{CC}}^{V}, \\
\left(U_{\mathrm{CL}}^{A}\right)_{\mathrm{c} .} & =-\frac{\lambda}{\kappa}\left(U_{\mathrm{CC}}^{A}\right)_{\mathrm{c} .}, \\
U_{\mathrm{LL}}^{V} & =\frac{\lambda^{2}}{\kappa^{2}} U_{\mathrm{CC}}^{V}, \\
\left(U_{\mathrm{LL}}^{A}\right)_{\mathrm{c} .} & =\frac{\lambda^{2}}{\kappa^{2}}\left(U_{\mathrm{CC}}^{A}\right)_{\mathrm{c} .},
\end{aligned}
$$

while the n.c. parts are

$$
\begin{aligned}
& \left(U_{\mathrm{CL}}^{A}\right)_{\text {n.c. }}=-\frac{\lambda \kappa}{\tau} G_{A}^{\prime 2}, \\
& \left(U_{\mathrm{LL}}^{A}\right)_{\text {n.c. }}=\frac{\kappa^{2}}{\tau} G_{A}^{\prime 2} .
\end{aligned}
$$

Finally, the transverse responses are given by

$$
\begin{aligned}
U_{T} & =U_{T}^{V}+U_{T}^{A}, \\
U_{T}^{V} & =2 \tau\left(2 G_{M}^{V}\right)^{2}+\frac{\left(2 G_{E}^{V}\right)^{2}+\tau\left(2 G_{M}^{V}\right)^{2}}{1+\tau} \Delta, \\
U_{T}^{A} & =2(1+\tau) G_{A}^{2}+G_{A}^{2} \Delta, \\
U_{T^{\prime}} & =2 G_{A}\left(2 G_{M}^{V}\right) \sqrt{\tau(1+\tau)}[1+\tilde{\Delta}],
\end{aligned}
$$

with

$$
\tilde{\Delta}=\sqrt{\frac{\tau}{1+\tau}} \frac{\xi_{F}\left(1-\psi^{2}\right)}{2 \kappa} .
$$

[1] D. A. Krakauer et al., Phys. Rev. C 45, 2450 (1992).

[2] B. Zeitnitz, Prog. Part. Nucl. Phys. 32, 351 (1994).

[3] B. E. Bodmann et al., Phys. Lett. B332, 251 (1994).

[4] M. Albert et al., Phys. Rev. C 51, 1065(R) (1995).

[5] C. Athanassopoulos, et al., Phys. Rev. C 56, 2806 (1997).

[6] C. Athanassopoulos, et al., Phys. Rev. C 58, 2489 (1998).

[7] A. Aguilar et al., Phys. Rev. D 64, 112007 (2001).

[8] L. B. Auerbach et al., Phys. Rev. C 66, 015501 (2002).

[9] E. Kolbe, K. Langanke, and P. Vogel, Phys. Rev. C 50, 2576 (1994).

[10] E. Kolbe, K. Langanke, and P. Vogel, Phys. Rev. C 62, 055502 (2000).

[11] E. Kolbe, K. Langanke, and P. Vogel, Nucl. Phys. A652, 91 (1999).

[12] A. C. Hayes and I. S. Towner, Phys. Rev. C 61, 044603 (2000).

[13] C. Volpe, N. Auerbach, G. Colo, T. Suzuki, and N. Van Giai, Phys. Rev. C 62, 015501 (2000).

[14] T. S. Kosmas and E. Oset, Phys. Rev. C 53, 1409 (1996).

[15] J. Nieves, J. E. Amaro, and M. Valverde, Phys. Rev. C 70, 055503 (2004).
[16] F. Krmpotić, A. Samana, and A. Mariano, Phys. Rev. C 71, 044319 (2005).

[17] Y. Fukuda et al. (Super-Kamiokande Collaboration), Phys. Rev. Lett. 81, 1562 (1998).

[18] M. H. Ahn et al. (K2K Collaboration), Phys. Rev. Lett. 90, 041801 (2003).

[19] E. Ables et al. (MINOS Collaboration), FERMILAB-Proposal0875 (1995).

[20] E. Church et al. (BooNe Collaboration), Fermilab-Proposal0898 (1997).

[21] I. Ambats et al. (NOvA Collaboration), Fermilab-Proposal-0929 (2004).

[22] D. Drakoulakos et al. (MINERvA Collaboration), "Proposal to perform a high-statistics neutrino scattering experience using a fine-grained detector in the NuMI beam," hep-ex/0405002.

[23] T. Kuramoto, M. Fukugita, Y. Kohyama, and K. Kubodera, Nucl. Phys. A512, 711 (1990).

[24] J. Engel, E. Kolbe, K. Langanke, and P. Vogel, Phys. Rev. D 48, 3048 (1993).

[25] G. D. Barr, T. K. Gaisser, P. Lipari, S. Robbins, and T. Stanev, 
Phys. Rev. D 70, 023006 (2004) and references therein.

[26] D. Drakoulakos et al. (MINERvA Collaboration), Proposal for FERMILAB experiment E938 (2004), hep-ex/0405002.

[27] J. E. Amaro, J. A. Caballero, T. W. Donnelly, and E. Moya de Guerra, Nucl. Phys. A611, 163 (1996).

[28] S. Jeschonnek and T. W. Donnelly, Phys. Rev. C 57, 2438 (1998).

[29] J. E. Amaro and T. W. Donnelly, Ann. Phys. (NY) 263, 56 (1998).

[30] J. E. Amaro and T. W. Donnelly, Nucl. Phys. A646, 187 (1999).

[31] J. M. Udias, J. A. Caballero, E. Moya de Guerra, J. E. Amaro, and T. W. Donnelly, Phys. Rev. Lett. 83, 5451 (1999).

[32] L. Alvarez-Ruso, M. B. Barbaro, T. W. Donnelly, and A. Molinari, Phys. Lett. B497, 214 (2001).

[33] J. E. Amaro and T. W. Donnelly, Nucl. Phys. A703, 541 (2002).

[34] M. Mazziotta, J. E. Amaro, and F. A. de Saavedra, Phys. Rev. C 65, 034602 (2002).

[35] F. K. Tabatabaei, J. E. Amaro, and J. A. Caballero, Phys. Rev. C 68, 034611 (2003).

[36] F. K. Tabatabaei, J. E. Amaro, and J. A. Caballero, Phys. Rev. C 69, 064607 (2004).

[37] J. E. Amaro, J. A. Caballero, T. W. Donnelly, and F. K. Tabatabaei, Nucl. Phys. A753, 189 (2005).

[38] J. E. Amaro, M. B. Barbaro, J. A. Caballero, T. W. Donnelly, and A. Molinari, Nucl. Phys. A643, 349 (1998).

[39] J. E. Amaro, M. B. Barbaro, J. A. Caballero, and F. K. Tabatabaei, Phys. Rev. C 68, 014604 (2003).

[40] J. E. Amaro, M. B. Barbaro, J. A. Caballero, T. W. Donnelly, and A. Molinari, Phys. Rep. 368, 317 (2002).

[41] W. M. Alberico et al., Nucl. Phys. A623, 471 (1997).

[42] W. M. Alberico et al., Phys. Lett. B438, 9 (1998).

[43] W. M. Alberico et al., Nucl. Phys. A651 277 (1999).

[44] C. Maieron, M. C. Martínez, J. A. Caballero, and J. M. Udías, Phys. Rev. C 68, 048501 (2003).

[45] A. Meucci, C. Giusti, and F. D. Pacati, Nucl. Phys. A739, 277 (2004); 744, 307 (2004).
[46] W. M. Alberico, A. Molinari, T. W. Donnelly, E. L. Kronenberg, and J. W. Van Orden, Phys. Rev. C 38, 1801 (1988).

[47] D. B. Day, J. S. McCarthy, T. W. Donnelly, and I. Sick, Annu. Rev. Nucl. Part. Sci. 40, 357 (1990).

[48] M. B. Barbaro, R. Cenni, A. De Pace, T. W. Donnelly, and A. Molinari, Nucl. Phys. A643, 137 (1998).

[49] T. W. Donnelly and I. Sick, Phys. Rev. Lett. 82, 3212 (1999).

[50] T. W. Donnelly and I. Sick, Phys. Rev. C 60, 065502 (1999).

[51] C. Maieron, T. W. Donnelly, and I. Sick, Phys. Rev. C 65, 025502 (2002).

[52] M. B. Barbaro, J. A. Caballero, T. W. Donnelly, and C. Maieron, Phys. Rev. C 69, 035502 (2004).

[53] J. E. Amaro, M. B. Barbaro, J. A. Caballero, T. W. Donnelly, A. Molinari, and I. Sick, Phys. Rev. C 71, 015501 (2005).

[54] T. W. Donnelly and R. D. Peccei, Phys. Rep. 50, 1 (1979).

[55] T. W. Donnelly, Prog. Part. Nucl. Phys. 13, 183 (1985).

[56] H. Kim, S. Schramm, and C. J. Horowitz, Phys. Rev. C 53, 2468 (1996).

[57] M. B. Barbaro, A. De Pace, T. W. Donnelly, A. Molinari, and M. J. Musolf, Phys. Rev. C 54, 1954 (1996).

[58] J. D. Bjorken and S. D. Drell, Relativistic Quantum Mechanics (McGraw-Hill, New York, 1964).

[59] S. Galster et al., Nucl. Phys. B32, 221 (1972).

[60] J. E. Amaro, C. Maieron, J. Nieves, and M. Valverde, Eur. Phys. J. A, in press.

[61] R. Rosenfelder, Phys. Lett. B79, 15 (1978).

[62] R. D. Smith and J. Wambach, Phys. Rev. C 38, 100 (1988).

[63] J. E. Amaro, G. Co', and A. M. Lallena, Ann. Phys. (NY) 221, 306 (1993).

[64] J. E. Amaro, G. Co', and A. M. Lallena, Nucl. Phys. A578, 365 (1994).

[65] A. R. Edmonds, Angular Momentum in Quantum Mechanics (Princeton University Press, Princeton, NJ, 1974).

[66] M. Rotenberg, R. Bivins, N. Metropolis, and J. K. Wooten, Jr., The 3-j and 6-j Symbols (MIT, Cambridge, MA, 1959). 